\title{
The role of the ferroptosis-related gene in the immune activity and prognosis of sepsis
}

Wei Dai ${ }^{1,2}$, Tian Yü ${ }^{3,4}$, Deqiang Luo ${ }^{1,2}$, Qian Xie ${ }^{1,2}$, Fen Liü ${ }^{1,2}$, Qiang Shao ${ }^{1,2}$, Nin Zhao ${ }^{1,2}$, Ping Zheng ${ }^{2,5}$, Kejian Qian ${ }^{1,2}$

${ }^{1}$ Department of Intensive Care Unit, The First Affiliated Hospital of Nanchang University, Dong Lake District, Nanchang, Jiangxi Province, China

${ }^{2}$ Medical Innovation Center, The First Affiliated Hospital of Nanchang University,

Nanchang, Jiangxi Province, China

${ }^{3}$ Graduate School, Chinese Academy of Medical Sciences and Peking Union Medical College, Beijing, China

${ }^{4}$ Department of General Surgery, Peking Union Medical College Hospital, Beijing, China

${ }^{5}$ Department of Key Laboratory, Shanghai Pudong New area People's Hospital, Shanghai, China

Submitted: 23 November 2021; Accepted: 1 January 2022

Online publication: 2 January 2022

Arch Med Sci

DOI: https://doi.org/10.5114/aoms/145473

Copyright @ 2022 Termedia \& Banach

\section{Abstract}

Introduction: Sepsis is a leading cause of mortality in intensive care units worldwide. Ferroptosis, a form of regulated cell death dependent on iron, has been proven to be altered during sepsis, including increased iron transport and uptake into cells and decreased iron export. A better understanding of the role of ferroptosis in sepsis should expedite the identification of biomarkers for prognostic evaluation and therapeutic interventions.

Material and methods: We used the mRNA expression profiles of sepsis patients from Gene Expression Omnibus (GEO) to analyze the expression level of ferroptosis-related genes and construct molecular subtypes.

Results: Two distinct ferroptosis patterns were determined, and the overall survival of the two clusters was highly significantly different. Gene comparison analysis was performed on these two groups, and there were a total of 106 differentially expressed genes (DEGs). Pathway enrichment analysis of these genes showed that ferroptosis and immune-related pathways were enriched, suggesting that immune pathways might be critically involved in sepsis. To systematically predict the prognosis of sepsis, we constructed a nomogram model; the calibration plot nomogram showed excellent concordance for the 7-, 14-, and 28-day predicted and actual overall survival probabilities. Finally, the results of bioinformatics analysis were validated in animal and cell models

Conclusions: In this study, we construct a ferroptosis-related nomogram that can be used for prognostic prediction in sepsis. In addition, we revealed that ferroptosis played a non-negligible role in immune cell infiltration and guiding more effective immunotherapy strategies.

Key words: sepsis, ferroptosis, immune cell infiltration, prognostic evaluation.

\section{Introduction}

Sepsis is a life-threatening organ dysfunction caused by a disorder of the body's response to infection [1]. Sepsis has become one of the top

\author{
Corresponding author: \\ Kejian Qian \\ Department of \\ Intensive Care Unit \\ The First Affiliated \\ Hospital of \\ Nanchang University \\ Medical Innovation Center \\ 17 Yongwaizheng St \\ Dong Lake District \\ Nanchang 330000 \\ Jiangxi Province, China \\ E-mail: ndyfyicu@email.ncu. \\ edu.cn
}


10 causes of death worldwide, and is a common cause of complications and death among patients in intensive care units (ICU) [2]. For many years, sepsis was thought to result from a highly inflammatory immune response in the host to infection [3]. Cytokines produced by severe inflammatory responses activate neutrophils and cause excessive production of reactive oxygen species (ROS), which can lead to tissue and organ damage and further development of organ dysfunction and failure [4]. However, the mechanism and biological role of sepsis induction are still not clear. Therefore, it is necessary to explore the role of immunity in sepsis from the perspective of molecular genetics [5].

In recent years, more and more attention has been paid to the role of abnormal metabolism of trace elements in sepsis [6-8]. Iron is an important trace element that is needed for many basic processes, including DNA synthesis, energy production and immune function [9]. Ferroptosis is a novel iron-dependent mode of cell death discovered in recent years; its main characteristics are iron metabolism changes and lipid peroxidation [10]. Ferroptosis is a death pathway different from necrosis, apoptosis and other typical characteristics of cell death [11]. The ultrastructure of ferroptosis showed that mitochondria became smaller, mitochondria cristae disappeared, membrane density increased, outer membrane rupture occurred and cell size decreased [11]. The main mechanism in volved iron divalent induced intracellular lipid peroxidation and decreased expression of glutathione peroxidase (GPX) and glutathione peroxidase 4 (GPX4) [9]. Recent studies have shown that ferroptosis in sepsis can be alleviated by improving oxidative stress [12-14]. In this study, we explored the molecular mechanism of ferroptosis during sepsis, in order to provide ideas for prevention and treatment of sepsis.

The pathological mechanism of sepsis is complex, and many cell death modes are involved [11, $15,16]$. With the development of research on the mechanism of ferroptosis over the last decade, ferroptosis has been proved to play a role in the pathogenesis of diseases caused by microbial infection $[12,17,18]$. A large number of studies have shown that ferroptosis plays an important role in inflammation. In addition, immune mechanisms can also regulate the sensitivity of cells to ferroptosis [12].

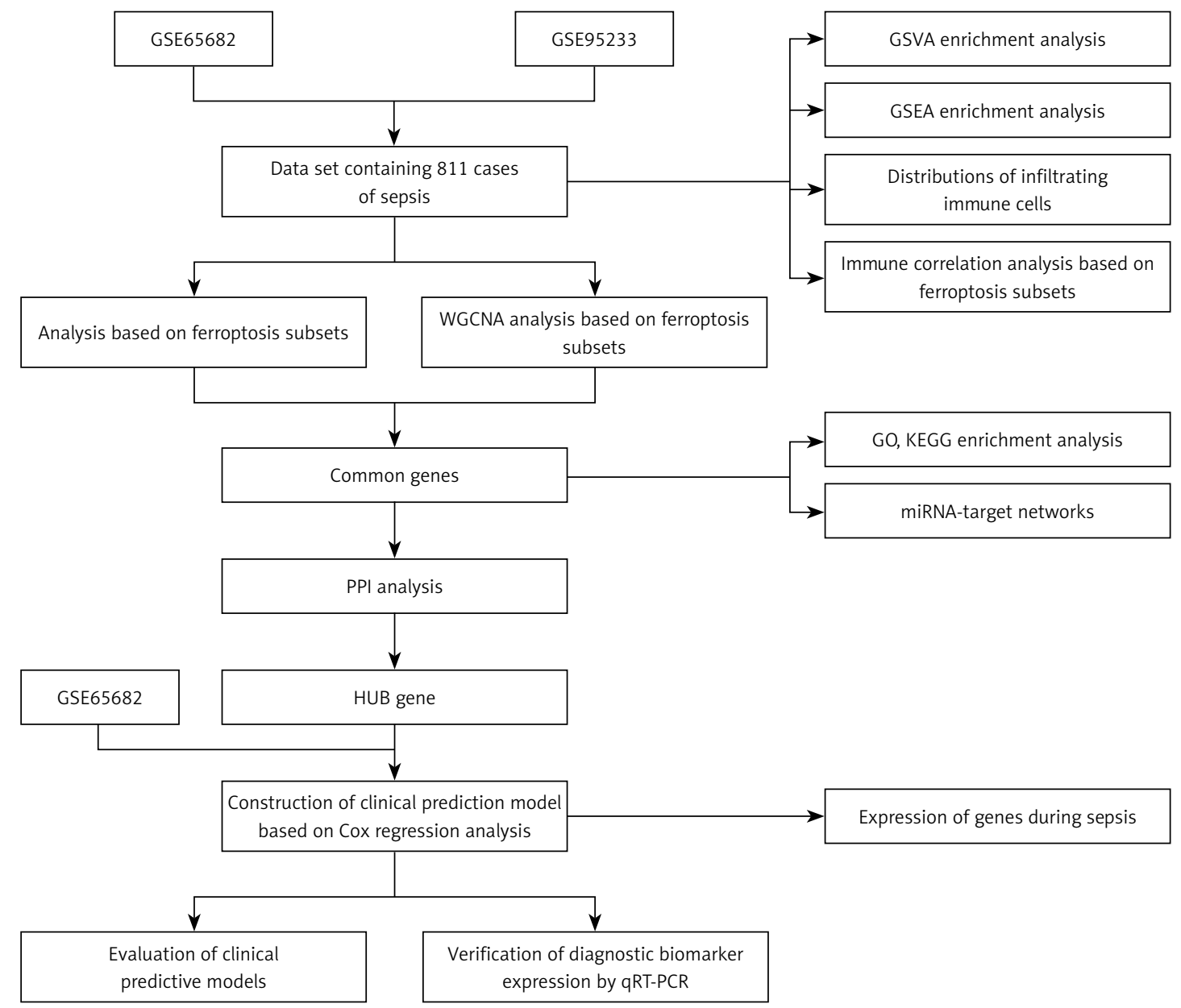

Figure 1. Flow chart of the overall analysis of the biological characteristics of sepsis 
Many studies have investigated the underlying mechanisms of the occurrence and development of various diseases from the perspective of ferroptosis, aiming to construct prognostic models based on bioinformatics analysis of ferroptosis $[9,10,16,18]$. With the development of high-throughput sequencing technology, many studies have successfully constructed prognostic models based on ferroptosis-related genes [19-21]. It shows potential clinical significance for prognostic prediction and development of molecular targeted drugs [22]. However, sepsis, the most common disease in the ICU, has no such prognostic model. Here, in this study, we develop a prognostic model based on ferroptosis-related genes and explore its prognostic effect on sepsis patients, and we also explore its association with immune infiltration.

\section{Material and methods}

\section{Sepsis datasets source and preprocessing}

The flow chart of the overall study is shown in Figure 1. We searched GEO (https://www.ncbi. nlm.nih.gov/geo/) for experiments studying sepsis in human whole blood samples and found 2 datasets (GEO accession numbers: GSE65682, GSE95233) [23, 24]. We downloaded the data using the GEOquery package of R software (version 4.1.0, http://r-project.org/) [25]. GSE65682 was based on the GPL13667[HG-U219] Affymetrix Human Genome U219 Array platform, and the dataset contained 802 blood samples, including 760 sepsis cases and 42 healthy controls. GSE95233 was based on the GPL570[HG-U133_plus_2] Affymetrix Human Genome U133 Plus 2.0 Array platform, and the data set included 51 sepsis patients and 22 control patients, a total of 73 patients. Blood samples from sepsis patients on the first day of ICU admission were included in the study. The expression profile data of sepsis samples from the above two data sets were combined into one data set, and data cleaning operations such as batch removal, standardized processing and annotation probe were carried out. The clinical information of dataset GSE65682 was extracted for clinical analysis. The next step was to obtain genes related to ferroptosis. Firstly, 304 genes related to ferroptosis were searched in the genecards database (https://www.genecards.org/) using ferroptosis as the keyword. Then a total of 288 ferroptosis genes related to Driver, Suppressor and Marker were obtained from the FerrDb database (http://www.zhounan.org/ferrdb/). Finally, a total of 470 ferroptosis genes were obtained after the combination of the two datasets and the removal of duplicate genes.

\section{Consensus clustering for ferroptosis related} genes

Consensus clustering analysis was applied to identify distinct ferroptosis patterns based on the expression of ferroptosis related genes. We used the ConsensusClusterPlus package to perform the above steps; cluster 1 and cluster 2 are classified according to the results [26]. The correctness of the classification was verified by principal component analysis (PCA) of the mRNA expression profile based on sepsis. Survival analysis was performed using the clinical data of GSE65682 to analyze whether the classification of ferroptosis subtypes was clinically significant. The "limma" $R$ package was used to analyze the differentially expressed genes (DEGs) between the two clusters with a false discovery rate $(F D R)<0.05$ and logarithmic fold change (LFC) $>0.3$ [27]. The ggplot2 $R$ package was used to draw the volcano map and heat map of differential genes to show the expression of DEGs [28].

\section{Evaluation of immune cell infiltration}

We used CIBERSORT, an analytical algorithm that deconvolutes bulk samples with a minimal representation for each immune cell type using support vector regression based on a set of reference gene expression values [29]. CIBERSORT analyzes RNA expression data to evaluate the abundance of different immune cell subtypes in each sample, to examine immune cell types of sepsis and to estimate the proportion of infiltrating immune cells. Only samples with a CIBERSORT output of $p<0.05$ were considered worthy of further analysis. The ggplot2 $\mathrm{R}$ package was used to visualize the expression differences of 22 immune cells between cluster 1 and cluster2. The Corrplot $\mathrm{R}$ package was used to draw correlation heat maps to visualize the correlation of 22 immune cells' infiltration.

\section{Gene set variation analysis (GSVA) and gene set enrichment analysis (GSEA)}

To investigate the differences in biological processes between cluster 1 and cluster2, we performed GSVA enrichment analysis using "GSVA" $R$ packages [30]. GSVA, in a non-parametric and unsupervised method, is commonly employed for estimating the variation in pathway and biological process activity in the samples of an expression dataset. The gene sets of "c2.all.v7.4.symbols. gmt" were downloaded for running GSVA analysis. The rank-based approach GSEA was applied to evaluate the enrichment of ferroptosis genes. An adjusted $p$-value less than 0.05 was considered as statistically significant. 


\section{Weighted gene co-expression network analysis (WGCNA) based on ferroptosis cluster}

The WGCNA R package was applied to process data [31]. Firstly, the soft threshold value of network construction is selected, and the adjacency matrix is the continuous value between 0 and 1 , so that the constructed network accords with the power-law distribution and is closer to the real biological network state. Secondly, a scale-free network was constructed by using the function of block modules, and then the co-expression modules were identified by block partitioning analysis, so that genes with similar expression patterns were grouped. These modules are defined by using a dynamic tree cutting algorithm to cut the component branches of the cluster tree and assign different colors for visualization. All modules are summarized by modular characteristic genes (ME), the most important major component of each module, which are calculated as synthetic genes representing the expression profile of all genes in a given module.

\section{Functional enrichment analysis}

We used the "clusterProfiler" R package to perform Gene Ontology (GO) and Kyoto Encyclopedia of Genes and Genomes (KEGG) analyses of DEGs between cluster1 and cluster2 (FDR < 0.05) [32]. $\mathrm{GO}$ analysis is a common method for conducting large-scale functional enrichment studies, including biological processes (BP), molecular functions (MF), and cellular components (CC).

\section{Protein-protein interaction (PPI) network construction}

In this study, we used the STRING database (http://string-db.org, version 11.09) to predict protein-protein interaction (PPI) and selected genes with a database score greater than 0.4 to construct PPI networks. Cytoscape software (v3.7.2) was used to perform network visualization, and the cytoHubba plug-in was used to screen the top 20 hub genes according to the score.

\section{Constructing regulatory network}

The associations between hub genes and miRNAs were analyzed in this study after identifying the potential mRNAs and miRNAs through miRTarBase databases (http://mirtarbase.mbc.nctu.edu. tw). An integrating network of regulatory connections of mRNAs and miRNAs was constructed with the R package multiMiR [32]. Cytoscape software (v3.7.2) was used to perform network visualization.

\section{Construction of a predictive nomogram}

We used Cox regression to detect the prognosis-associated ferroptosis-related gene signature. The Survival package and SurvMiner package were used to perform univariate Cox regression analysis on GSE65682. The risk factors were included in the multivariate Cox regression analysis and the regression model was established. A nomogram was established based on the multivariate Cox model to predict the 28-day survival of sepsis patients. Finally, the correction curve was used to evaluate the accuracy and resolution of the nomogram.

\section{Construction of animal and cell models}

The healthy 8-week-old male rats used in this study were purchased from the Animal Laboratory of Nanchang University. The rat were reared in sterile cages with humidity of $45-55 \%$ and a light/dark cycle of $12 \mathrm{~h}$. The rats were adaptively reared for 1 week before animal experiments. All animal experiments were conducted in accordance with the Guidelines for The Use of Experimental Animal Care approved by the Ethics Committee of Nanchang University (Approval No.: 81960346). Next, the rat sepsis model was established by cecal ligation and puncture (CLP) and venous blood samples were obtained. The rats were anesthetized with sodium pentobarbital (50 mg/kg, intraperitoneal injection), and the cecum was exposed by cutting $1.5-2 \mathrm{~cm}$ along the midline of the abdomen. After stripping the mesentery, except for the sham group, half of the cecum was ligated with line 4. Except for the rat in the sham operation group, the cecum was punctured at $1 \mathrm{~cm}$ from the distal end of the ligation with a no. 21 sterile needle and the wound was sutured. For fluid resuscitation, each rat received $1 \mathrm{ml}$ of preheated saline $\left(37^{\circ} \mathrm{C}\right)$. Analgesic treatment: Rats were injected with buprenorphine $(0.05 \mathrm{mg} / \mathrm{kg}$, subcutaneously) every $6 \mathrm{~h}$ for 2 consecutive days.

The NR8383 cell line (rat lung macrophage) and RLE-6TN cell line (rat lung epithelial type II cells) were inoculated in 6-well plates with $2 \mathrm{ml}$ of medium per well at a density of 106 . When the cells grew to $50 \%$, the cells were stimulated with $1 \mu \mathrm{g} /$ $\mathrm{ml}$ LPS, and collected $2 \mathrm{~h}$ and $9 \mathrm{~h}$ later, respectively, to establish the sepsis cell model.

\section{qRT-PCR verification}

Total RNA was extracted with TRIzol reagent (Takara, Dalian, China). Prime-script RTase (Takara) was used for reverse transcription. With the help of premixed ex-Taq (Takara), gene expression levels were determined by GPCR and normalized to GAPDH expression levels. Expression levels were calculated using the $2^{-\Delta C T}$ method. 


\section{Drug sensitivity analysis} (www.cancerrxgene.org) cell line and TCGA gene, the pRRophetic algorithm was used to construct a ridge regression model to predict drug $\mathrm{IC}_{50}$, and drugs with significant differences between groups were screened out [33]. The top 20 drugs were selected for visualization.

\section{Statistical analysis}

All of the analyses were performed using $R$ with a two-sided significance threshold of $p<0.05$. For the comparison of the two groups of continuous variables, the statistical significance of the normally distributed variables was estimated using the independent Student $t$ test, and the differenc-
According to the expression profile of the GDSC

es between the non-normally distributed variables were analyzed using the Mann-Whitney $U$ test (i.e. Wilcoxon rank-sum test). The $\chi^{2}$ test or Fisher's exact test was used to compare and analyze the statistical significance between the two groups of categorical variables.

\section{Results}

\section{Subtype construction of sepsis based on ferroptosis}

Firstly, ConsensusClusterPlus software was used to construct subtypes based on ferroptosis gene expression profiles to explore the biological differences among different ferroptosis subtypes of sepsis. The classification is reliable and stable when $k=2$ (Figures $2 \mathrm{~A}-\mathrm{C}$ ). So we divided the

B

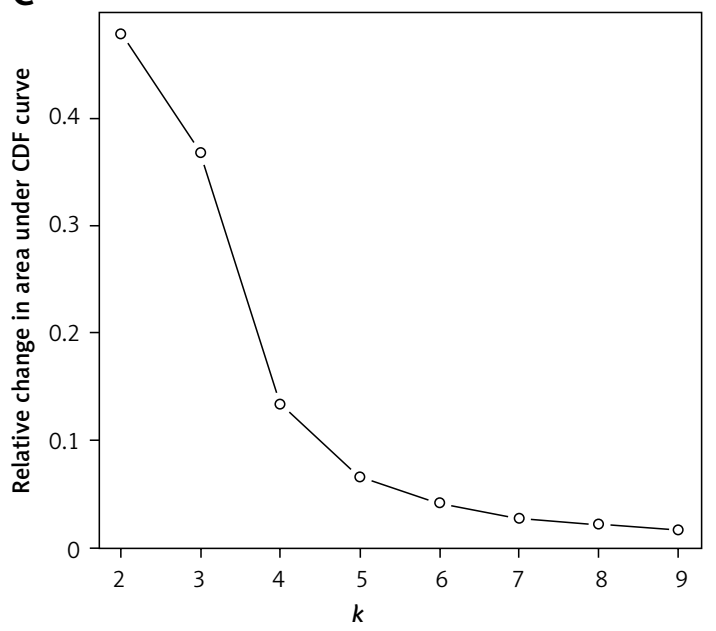

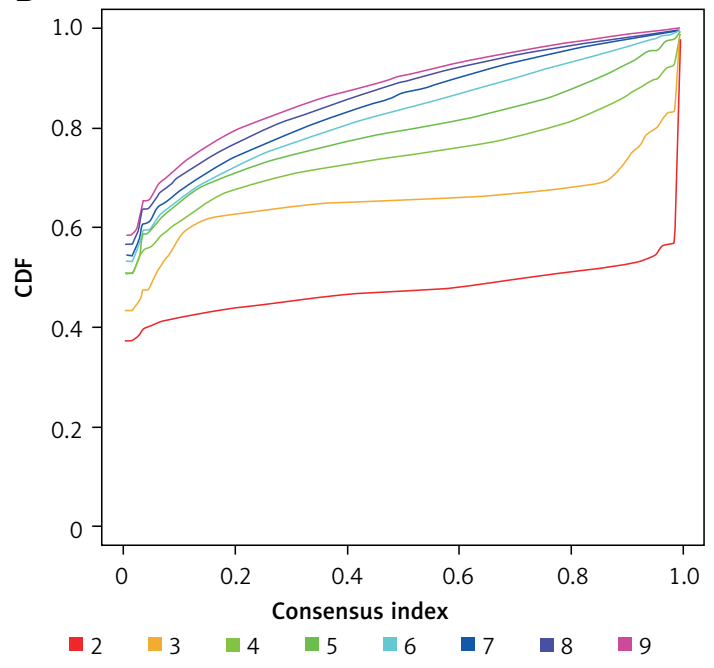

D
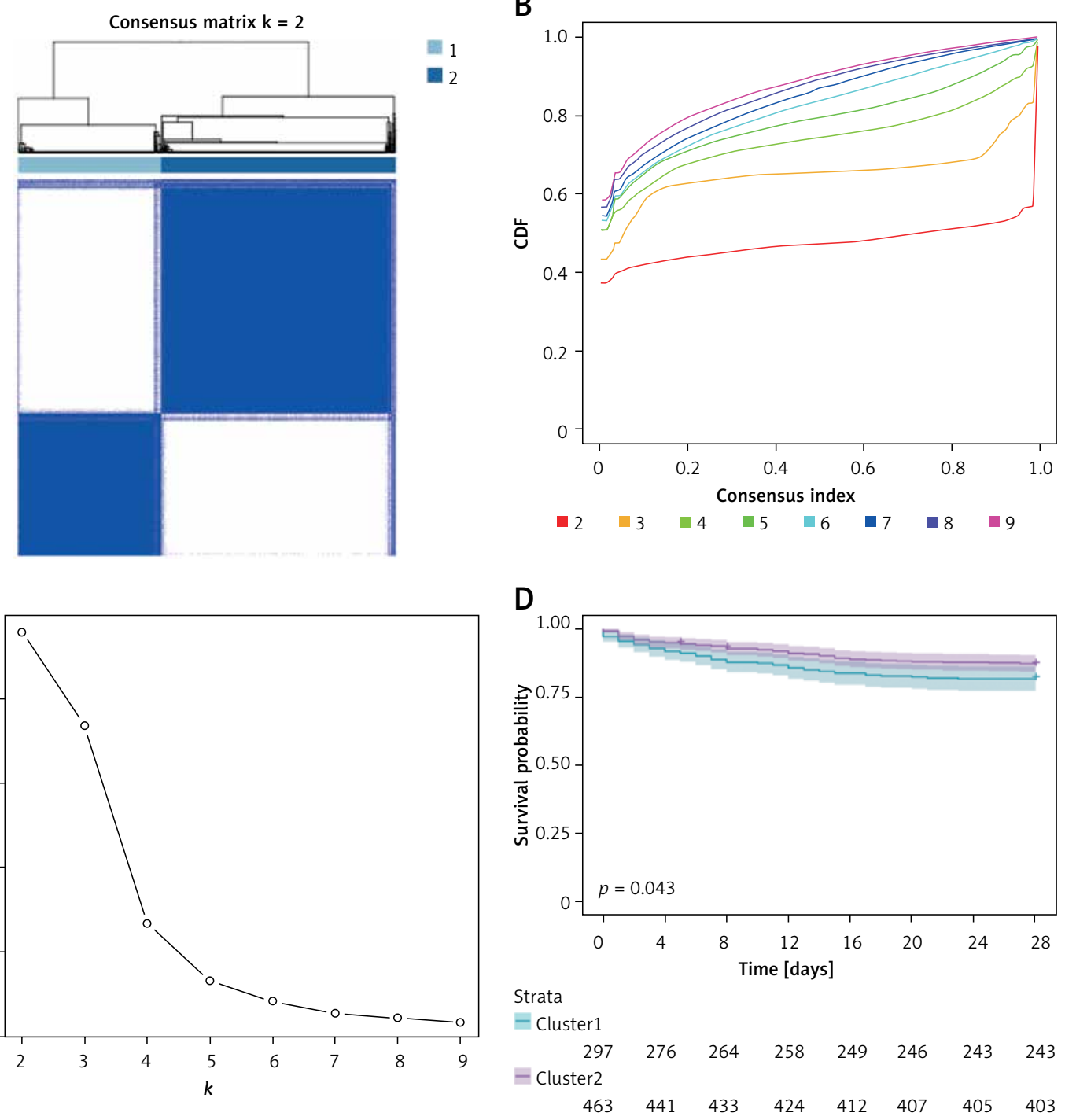

Figure 2. Characteristics of sepsis subtypes. A - The consensus matrix when $k=2$. Both rows and columns of the matrix represent samples. B - Consensus CDF; C - Delta area. D - Grouped by ferroptosis subtype, the difference in survival rate between the two groups $p=0.043$ 
samples into cluster 1 and cluster 2 . Further, we conducted survival analysis based on clinical data from the GSE65682 dataset, and found that there was a significant difference in the survival rate between cluster 1 and cluster 2 groups $(p<0.05)$, indicating that this grouping has clinical significance (Figure $2 \mathrm{D}$ ). Therefore, it is of practical significance for us to further explore the biological characteristics of ferroptosis.

PCA was used to further verify the correctness of classification of ferroptosis subtypes. It can be clearly seen that cluster 1 group and cluster 2 groups are clearly distinguished (Figure $3 \mathrm{~A}$ ). A differential expression analysis using $\mathrm{R}$ package lim- ma identified 106 differentially DEGs between the two groups, with a p. adjusted (FDR) value $<0.05$ and $\mid \log _{2} F C$ (fold change) $\mid>0.3$ as the cut-offs. Among these genes, 95 were downregulated and 11 were upregulated (Figures $3 \mathrm{~B}, \mathrm{C}$ ).

\section{Gene set variation analysis and gene set enrichment analysis}

"c2.all.v7.4.symbols.gmt" was selected as the reference gene set for GSVA analysis. There were significant differences in the following 10 gene sets based on ferroptosis subtype grouping: VALK AML_CLUSTER_7, VALK_AML_CLUSTER_8, XIE_ST_
A

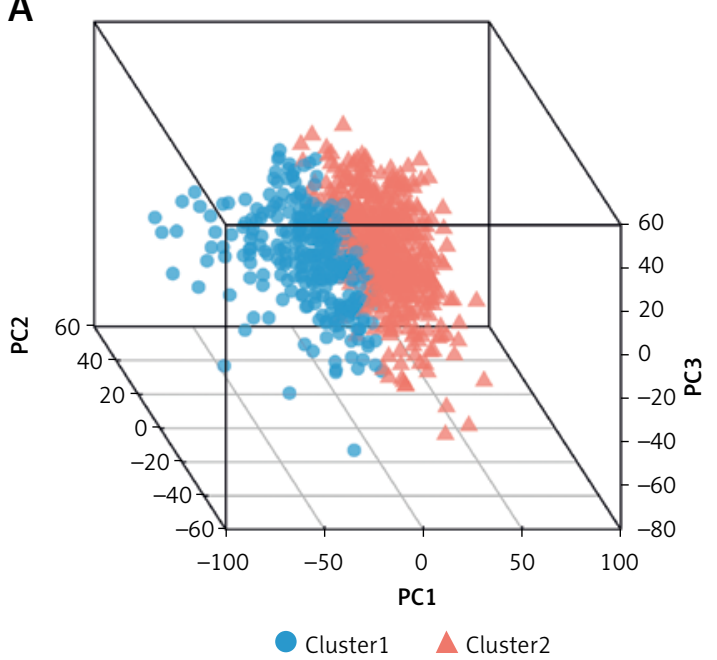

C

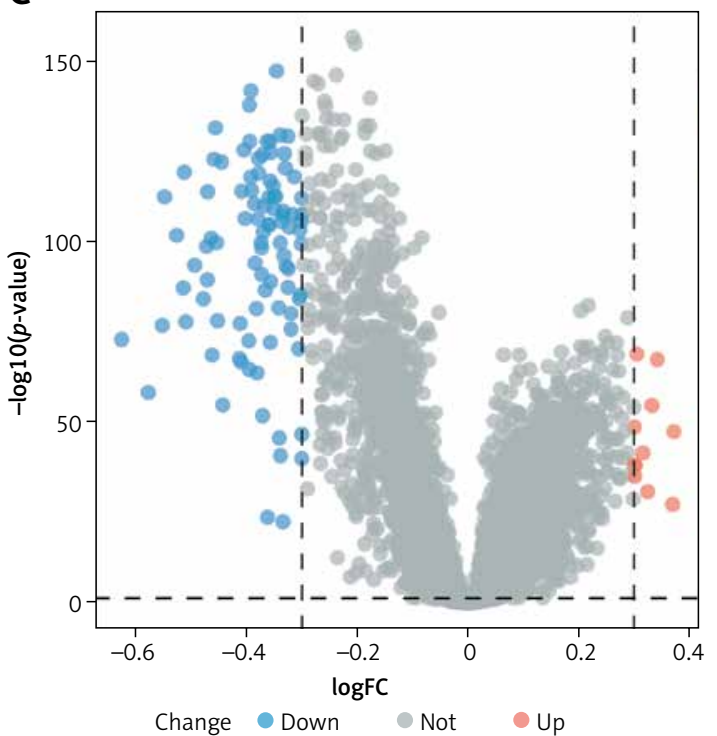

B

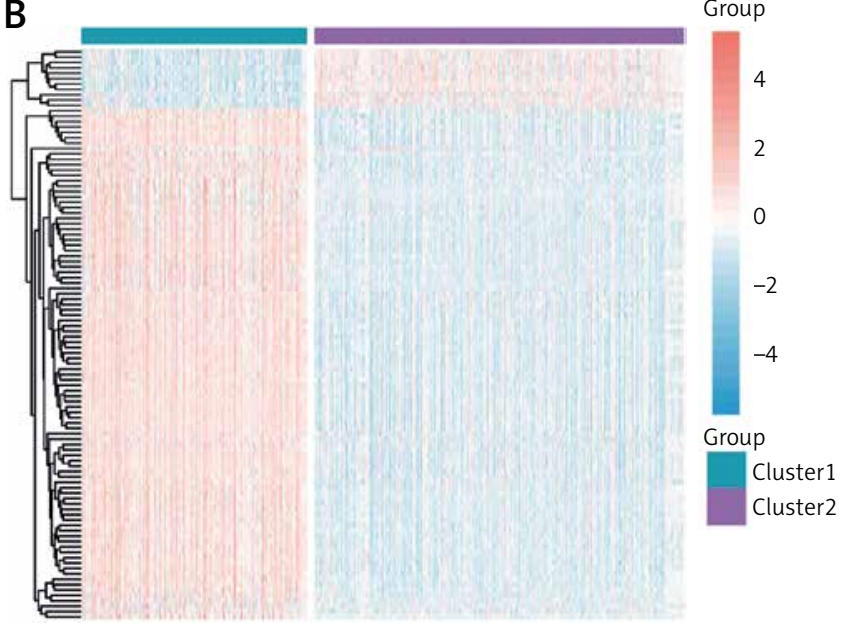

D

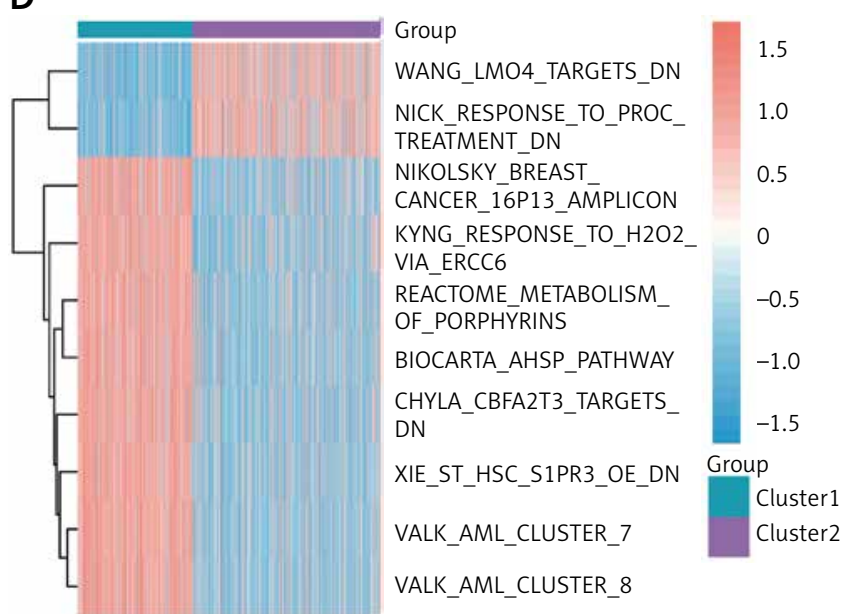

Figure 3. Difference analysis of ferroptosis subgroups and GSVA analysis. A - PCA analysis of ferroptosis subgroup, light blue is cluster1, light red is cluster2; B - The heat map of the different analyses of ferroptosis subgroups, light blue is cluster1, purple is cluster2, small blue squares represent low expression, and small red squares represent high expression; $\mathbf{C}$ - The volcano map of the different analyses of ferroptosis subgroups. The light blue point represents low expression, and the light red point represents high expression; D - According to GSVA analysis, light blue is cluster1, purple is cluster2, small blue squares represent low enrichment, and small red squares represent high enrichment 
Table I. GSVA enrichment analysis results

\begin{tabular}{|lccccc|}
\hline Pathway name & logFC & AveExpr & $t$ & $P$-value & B \\
\hline VALK_AML_CLUSTER_7 & 0.7660 & -0.0268 & 31.0918 & $1.37 \mathrm{E}-140$ & 310.5310 \\
\hline VALK_AML_CLUSTER_8 & 0.7967 & -0.0305 & 30.0983 & $1.94 \mathrm{E}-134$ & 296.4196 \\
\hline XIE_ST_HSC_S1PR3_OE_DN & 0.6401 & -0.0164 & 28.8664 & $8.53 \mathrm{E}-127$ & 278.8851 \\
\hline WANG_LMO4_TARGETS_DN & -0.3373 & 0.0109 & -28.8027 & $2.12 \mathrm{E}-126$ & 277.9779 \\
\hline CHYLA_CBFA2T3_TARGETS_DN & 0.3751 & -0.0067 & 28.3197 & $2.11 \mathrm{E}-123$ & 271.0997 \\
\hline KYNG_RESPONSE_TO_H2O2_VIA_ERCC6 & 0.5893 & -0.0084 & 28.2910 & $3.19 \mathrm{E}-123$ & 270.6908 \\
\hline REACTOME_METABOLISM_OF_PORPHYRINS & 0.5606 & -0.0137 & 28.0679 & $7.74 \mathrm{E}-122$ & 267.5122 \\
\hline BIOCARTA_AHSP_PATHWAY & 0.7278 & -0.0298 & 26.8280 & $3.81 \mathrm{E}-114$ & 249.8658 \\
\hline NICK_RESPONSE_TO_PROC_TREATMENT_DN & -0.4814 & 0.0131 & -26.0573 & $2.25 \mathrm{E}-109$ & 238.9187 \\
\hline NIKOLSKY_BREAST_CANCER_16P13_AMPLICON & 0.4482 & -0.0128 & 25.8906 & $2.41 \mathrm{E}-108$ & 236.5542 \\
\hline
\end{tabular}

HSC_S1PR3_OE_DN, WANG_LMO4_TARGETS_DN, CHYLA_CBFA2T3_TARGETS_DN, KYNG_RESPONSE_ TO H2Ö2 VIA ERCC6, REACTOME METABOLISM OF_PORPHYRINS, BIOCARTA_AHSP_PATHWAY, NICK_RESPONSE_TO_PROC_TREATMENT_DN and NIKOLSKY_BRDEAST_CANCER_16P13_AMPLICON. Most of these different gene sets were related to iron metabolism, inflammation, oxidative stress, immunity, blood diseases and other pathways (Figure $3 \mathrm{D}$, Table I).

In order to explore the functional enrichment of sepsis, GSEA analysis was performed on genes in the sepsis expression profile, with the reference gene set h.all.v7.4.entrez.gmt. The results showed that differential genes were significantly enriched in data sets HALLMARK HEME METABOLISM, HALLMARK_TNFA_SIGNALING_VIA_NFKB, HALLMARK_MYOGENESIS, HALLMARK_APICAL_JUNCTION, HALLMARK_XENOBIOTIC_METABOLISM, HALLMARK_COAGULATION, HALLMARK_PROTEIN_ SECRETION, HALLMARK INTERFERON GAMMA RESPONSE, HALLMARK_ANDROGEN_RESPONSE, HALLMARK_INFLAMMATORY_RESPONSE (Figures 4 A-I, Table II). It has an obvious correlation with inflammation, immunity, iron metabolism, coagulation, etc.

\section{Evaluation of immune cell infiltration}

Both GSEA and GEVA enrichment analysis found that sepsis was highly correlated with immunity. In order to further explore immune-related cell changes in sepsis patients, CIBERSORT was used to analyze the proportion of sepsis immune cells and construct 22 immune cell maps in sepsis samples (Figure $5 \mathrm{~A}$ ). In addition, we analyzed the correlation between immune cells (Figure $5 \mathrm{~B}$ ). The immune cell correlation matrix shows that the infiltration level of activated memory CD8+ T cells is highly correlated with neutrophils, indicating that these two types of immune cells play an important role in the course of sepsis (Figure $5 \mathrm{~B}$ ). In addition, we compared the differences of immune cell infiltration between cluster 1 and cluster2, and the results showed that $B$ cells naïve, $B$ cells memory, plasma cells, T cells CD8, T cells $\gamma \delta$, monocytes, dendritic cells resting, mast cells resting, mast cells activated and neutrophils were significantly different between the two clusters (Figure 5 C).

\section{WGCNA analysis based on ferroptosis subtypes}

The gene coexpression networks of the dataset were established via the WGCNA package (Figure $6 \mathrm{~A}$ ). To establish scale-free networks, the soft thresholding power was set at $\beta=5$ based on scale independence and mean connectivity (Figure 6 B). The dynamic tree cut package was used to generate a gene cluster dendrogram containing 20 co-expression models (Figure $6 \mathrm{C}$ ). The modules with the highest correlation with immune characteristics are brown $\left(r=0.76, p=7 \mathrm{e}^{-149}\right)$ (Figure 6 D). We intersected 106 DEGs grouped based on ferroptosis characteristics with 1251 genes in the brown module to obtain 94 intersection genes (Figure $6 \mathrm{E}$ ).

\section{Functional enrichment analysis}

In order to understand the biological functions and mechanisms between intersection genes and ferroptosis, we used GO enrichment analysis and KEGG pathway analysis on the intersection genes in this study. Erythrocyte development, Myeloid cell development, erythrocyte differentiation, cortical cytoskeleton, hemoglobin complex, cell cortex part, drug transmembrane transporter activity, 2 iron 2 sulfur cluster binding, Neutral amino acid transmembrane transporter activity and other biological function or characteristic were significantly enriched. However, when it comes to 

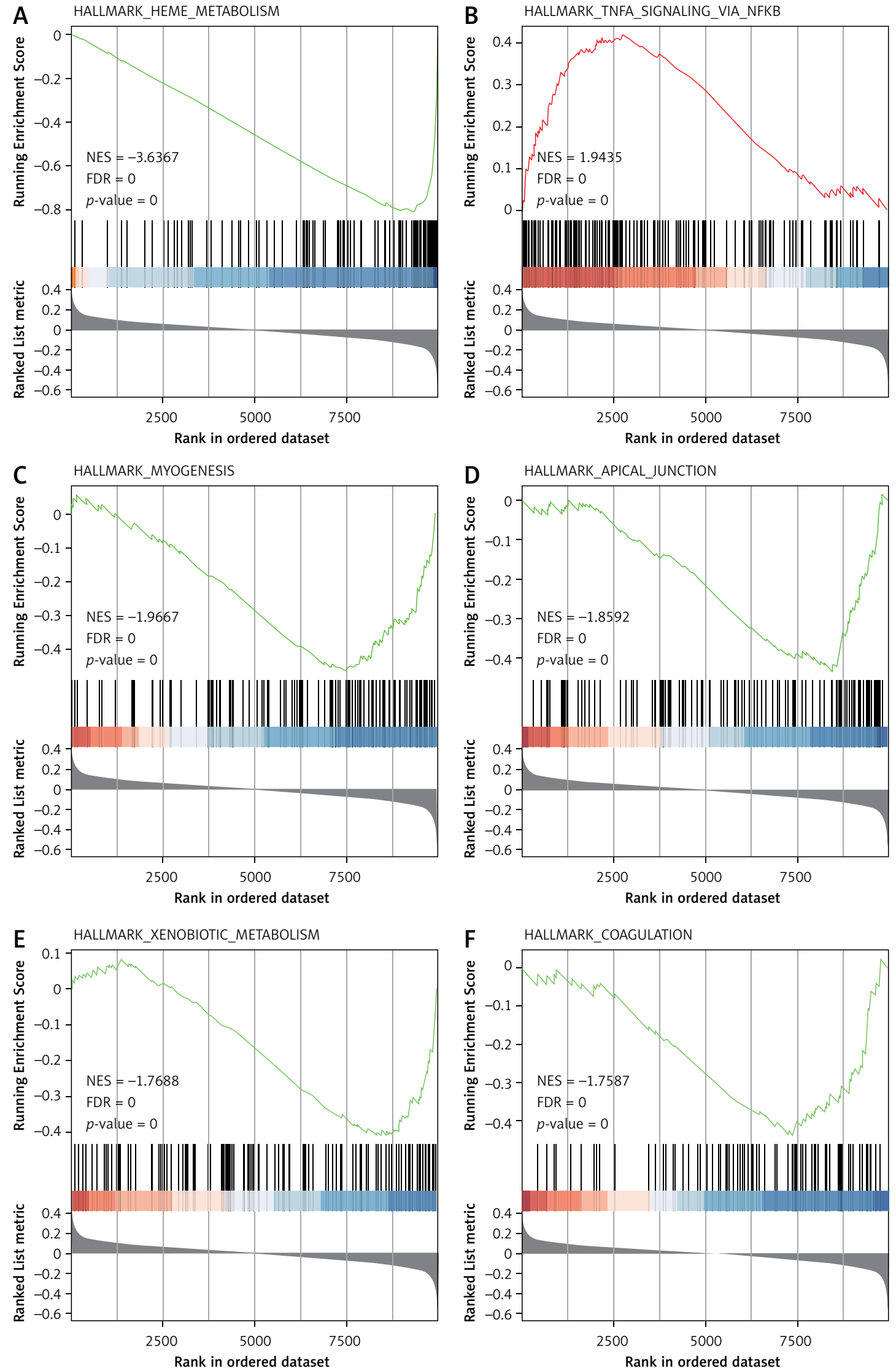

Figure 4. GSEA enrichment analysis. A-F - GSEA analysis of the most significant gene set 

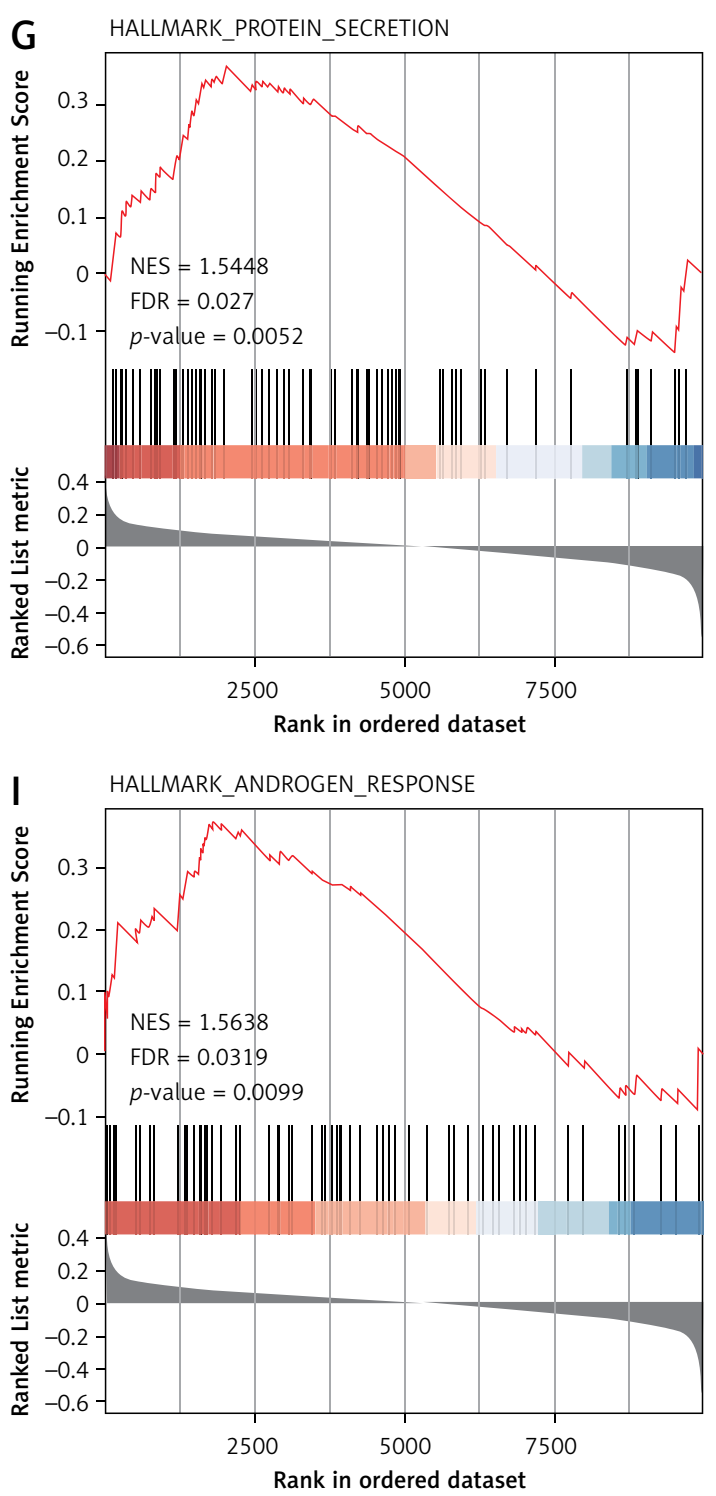

KEGG pathway enrichment analysis, only the malaria pathway was significantly enriched (Figure 7 A). In these inflammation, autophagy, apoptosis, iron metabolism and other related pathways, $\log F C$ is decreased, and the genes in them are also down-regulated (Figure $7 \mathrm{~B}$ ). Correlations between CISD2, SNCA, SESN3, FLCN, STK11, OPTN, PIP4K2A, SLC4A1, DMTN, EPB42, BPGM, NPRL3 and $\mathrm{GO}$ terms such as erythrocyte development, TORC1 signaling, negative regulation of TOR signaling, regulation of autophagy, and positive regulation of autophagy were observed (Figure 7 C).

\section{PPI and miRNA mRNA network construction}

In order to explore the functions of hubgenes transcribed and translated proteins, we used Cytoscape software to build a PPI network based on the STRING database. 62 proteins had close interactions with each other (Figure $7 \mathrm{D}$ ). The cytoHubba plug-in was used to screen the key genes,

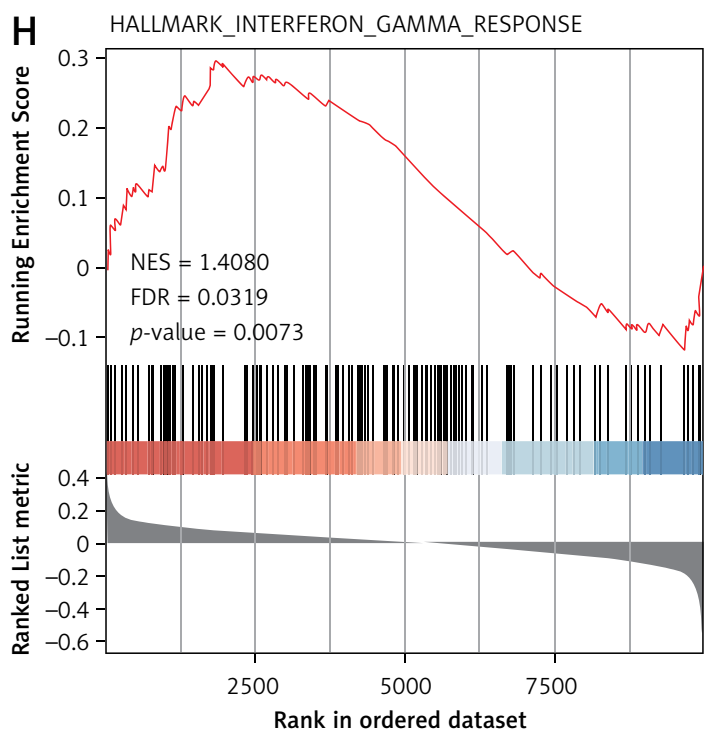

Figure 4. GSEA enrichment analysis. G-I - GSEA analysis of the most significant gene set

and the top 20 genes in cytoHubba were selected as hub genes (Figure $7 \mathrm{E}$ ). The top 20 genes were SLC4A1, EPB42, AHSP, DMTN, SNCA, FECH, KLF1, GYPB, GYPA, CA1, HBD, OSBP2, ANK1, TNS1, BPGM, SELENBP1, TMOD1, SPTB, FKBP8, and GMPR.

In order to further explore the upstream regulatory relationship, based on the miRTarBase V.8 database, the results of "Functional MTI" evidence were selected to predict the miRNA interacting with hub genes. The mRNA transcribed from the hub genes interacted with 330 miRNA (Figure 7 F).

\section{Construction of a predictive nomogram}

The clinical characteristic data and the expression data of 20 hub genes were extracted from GSE65682 datasets for univariate Cox regression analysis. The results showed that AHSP, DMTN, KLF1, GYPB, GYPA, CA1, HBD, BPGM, SPTB and GMPR had statistical significance $(p<0.05)$. We further conducted multivariate Cox regression 
Table II. GSEA enrichment analysis results

\begin{tabular}{|c|c|c|c|c|c|}
\hline Description & $\begin{array}{l}\text { Set- } \\
\text { Size }\end{array}$ & $\begin{array}{l}\text { Enrichment- } \\
\text { Score }\end{array}$ & NES & $P$-value & Leading_edge \\
\hline HALLMARK_HEME_METABOLISM & 176 & -0.8075 & -3.6367 & $1.00 \mathrm{E}-10$ & $\begin{array}{c}\text { tags }=58 \%, \text { list }=7 \%, \\
\text { signal }=55 \%\end{array}$ \\
\hline HALLMARK_TNFA_SIGNALING_VIA_NFKB & 150 & 0.4199 & 1.9435 & $1.56 \mathrm{E}-06$ & $\begin{array}{c}\text { tags }=46 \%, \text { list }=28 \%, \\
\text { signal }=34 \%\end{array}$ \\
\hline HALLMARK_MYOGENESIS & 115 & -0.4649 & -1.9668 & $9.42 \mathrm{E}-06$ & $\begin{array}{c}\text { tags }=50 \%, \text { list }=25 \%, \\
\text { signal }=38 \%\end{array}$ \\
\hline HALLMARK_APICAL_JUNCTION & 127 & -0.4325 & -1.8592 & $5.73 \mathrm{E}-05$ & $\begin{array}{c}\text { tags }=33 \%, \text { list }=15 \%, \\
\text { signal }=28 \%\end{array}$ \\
\hline HALLMARK_XENOBIOTIC_METABOLISM & 132 & -0.4069 & -1.7689 & $1.78 \mathrm{E}-04$ & $\begin{array}{c}\text { tags }=30 \%, \text { list }=15 \% \\
\text { signal }=26 \%\end{array}$ \\
\hline HALLMARK_COAGULATION & 83 & -0.4376 & -1.7587 & $6.77 \mathrm{E}-04$ & $\begin{array}{c}\text { tags }=45 \%, \text { list }=26 \% \\
\text { signal }=33 \%\end{array}$ \\
\hline HALLMARK_PROTEIN_SECRETION & 86 & 0.3686 & 1.5548 & $5.27 \mathrm{E}-03$ & $\begin{array}{c}\text { tags }=38 \%, \text { list }=20 \%, \\
\text { signal }=31 \%\end{array}$ \\
\hline HALLMARK_INTERFERON_GAMMA_RESPONSE & 175 & 0.2967 & 1.4080 & $7.36 \mathrm{E}-03$ & $\begin{array}{c}\text { tags }=26 \%, \text { list }=18 \%, \\
\text { signal }=21 \%\end{array}$ \\
\hline HALLMARK_ANDROGEN_RESPONSE & 76 & 0.3756 & 1.5638 & $7.81 \mathrm{E}-03$ & $\begin{array}{c}\text { tags }=34 \%, \text { list }=18 \%, \\
\text { signal }=28 \%\end{array}$ \\
\hline HALLMARK_INFLAMMATORY_RESPONSE & 149 & 0.3022 & 1.3985 & $9.95 \mathrm{E}-03$ & $\begin{array}{c}\text { tags }=48 \%, \text { list }=33 \% \\
\text { signal }=33 \%\end{array}$ \\
\hline
\end{tabular}

analysis on these genes and constructed a multivariate Cox regression model, which was visualized by a forest map (Figure $8 \mathrm{~A}$, Table IV). Through the forest plot of Cox regression, we can see that genes such as AHSP, GYPA, CA1, and BPGM may have important value for the prognosis of sepsis (Figure $8 \mathrm{~A}$ ). In addition, a nomogram was constructed using this model to predict 7-day, 14-day, and 28-day survival for patients with sepsis (Figure $8 \mathrm{~B}$ ). We further evaluated the predictive power of the line chart. The c-index of the line graph is 0.68 , indicating that it has high confidence. The calibration curves of days 7, 14 and 28 were consistent (Figures $8 \mathrm{C}-\mathrm{E}$ ), indicating that the nomogram has good accuracy and resolution.

\section{Experimental validation}

AHSP, DMTN, KLF1, GYPB, GYPA, CA1, HBD, BPGM, SPTB and GMPR were included in the previous multivariate Cox regression model construction. We further verified the expression of these genes by constructing septic animal and cell models. We performed verification in CPL animal

A

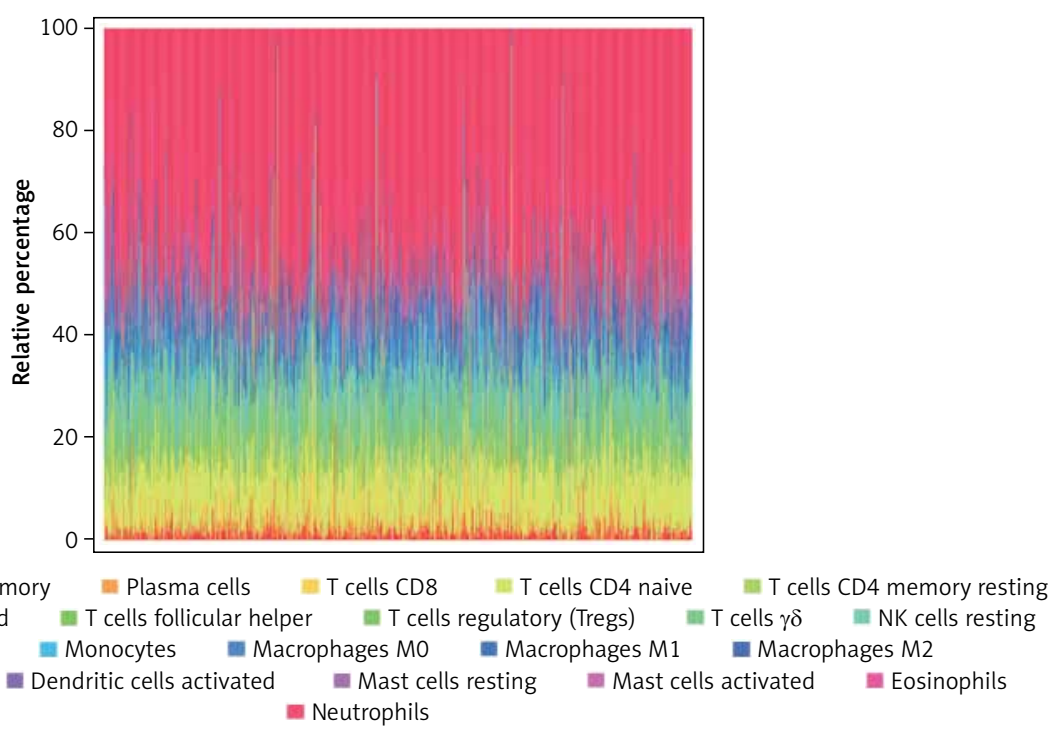

Figure 5. Immune cell infiltration and immune correlation analysis. A - Barplot of the ratio of 22 kinds of immune cells in sepsis samples 
B

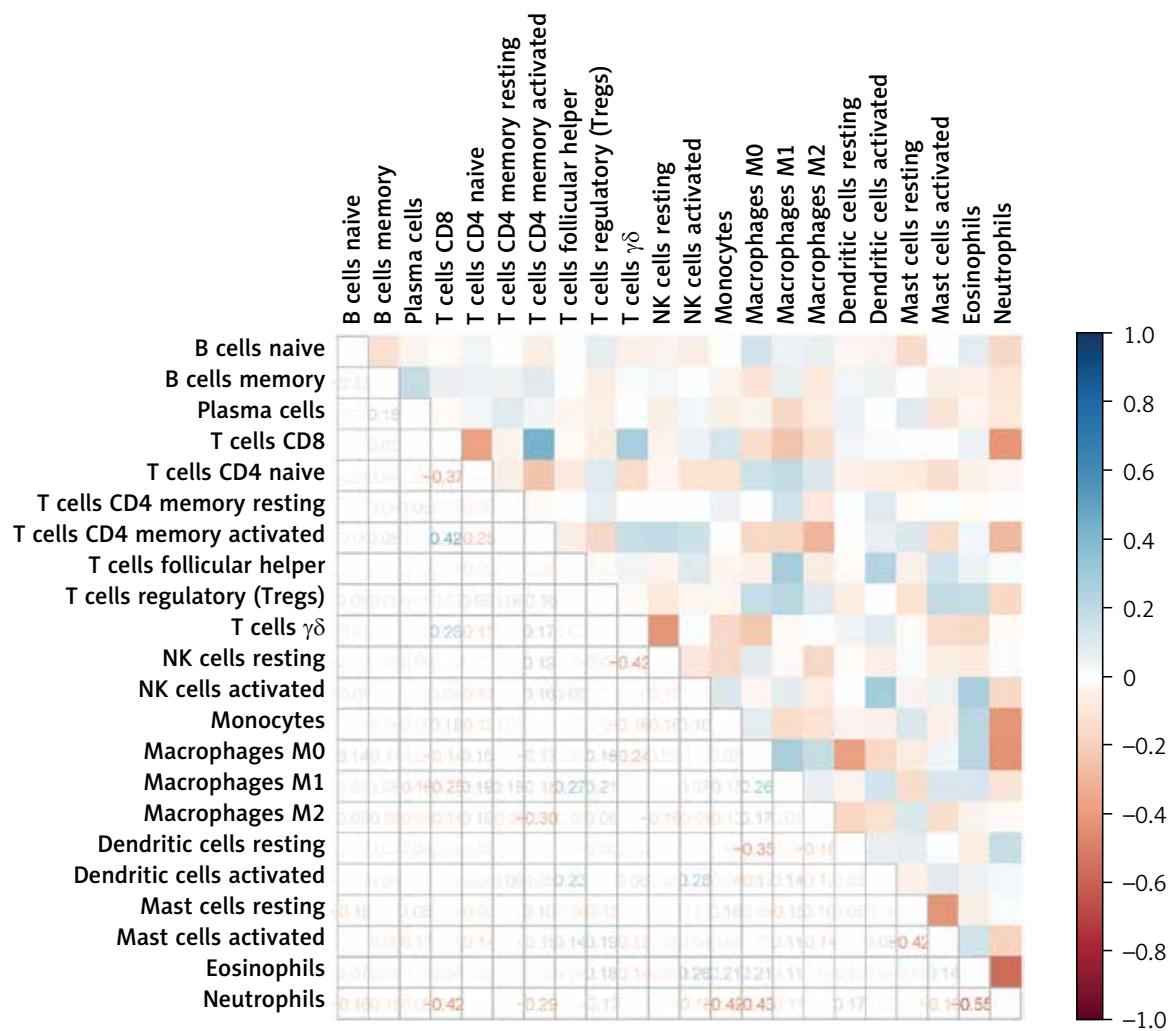

C

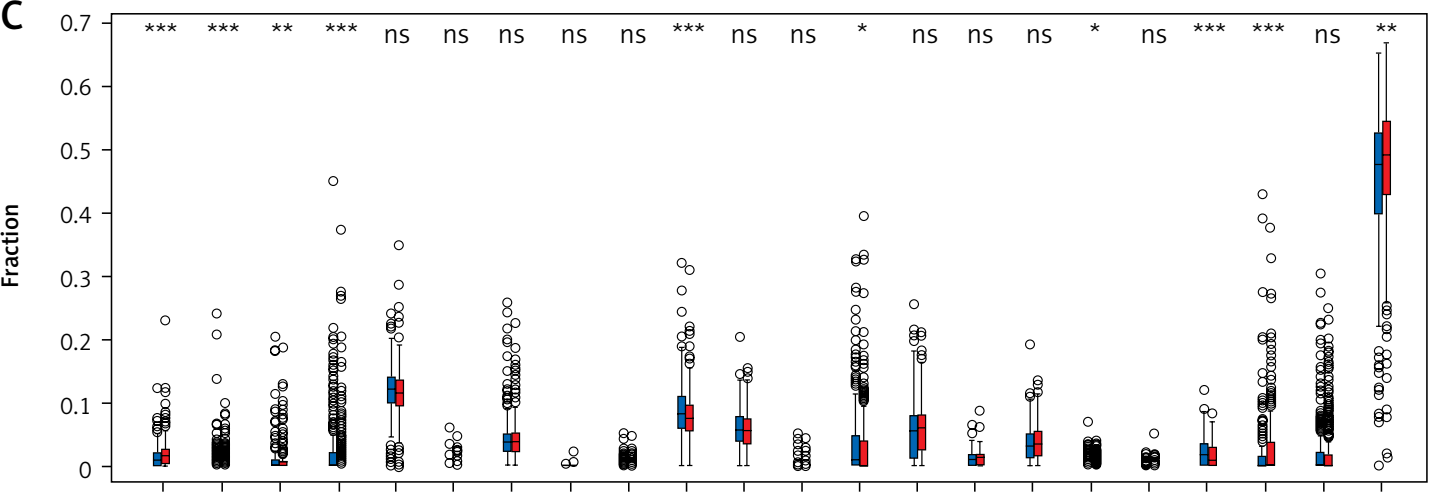

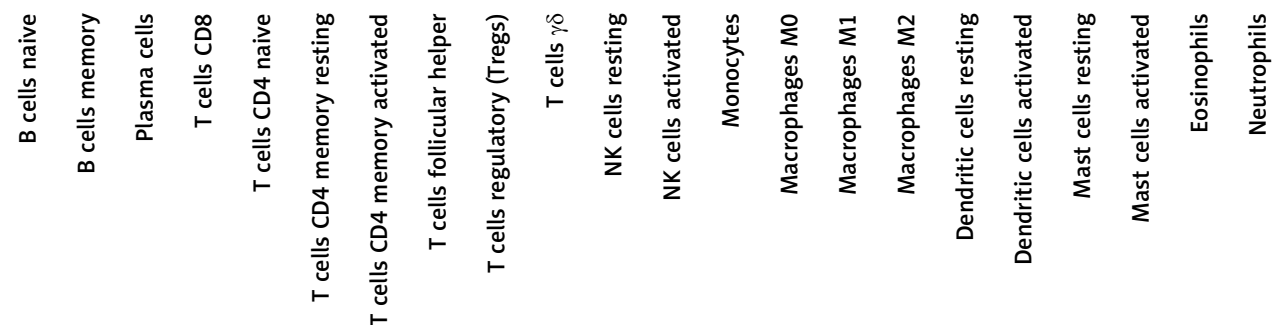

Figure 5. Cont. B - heat map of 22 kinds of immune cell infiltration; blue means positive correlation, red means negative correlation, the darker the color, the stronger the correlation; $\mathbf{C}$ - immune cells In the subtypes related to ferroptosis

models and cell models. We found that the expression of BPGM in the sepsis model was significantly higher than that in the control group, and the results were statistically significantly different (Figure $9 \mathrm{~A}$ ). In addition, consistent results were obtained in the two cell models MR8383 and RLE cell lines (Figure $9 \mathrm{~A}$ ). This proves that the expression of BPGM will increase significantly during the course of sepsis. Subsequently, we further verified several other genes included in the model, such as GYPA, AHSP, CA1, GMPR, SPTB, KLF1, HBG, and DMTN, and obtained consistent results. Since GYPB was not expressed in rat, other genes were used for subsequent experimental verification. The results showed that the expression level in the blood of sepsis animals was significantly 

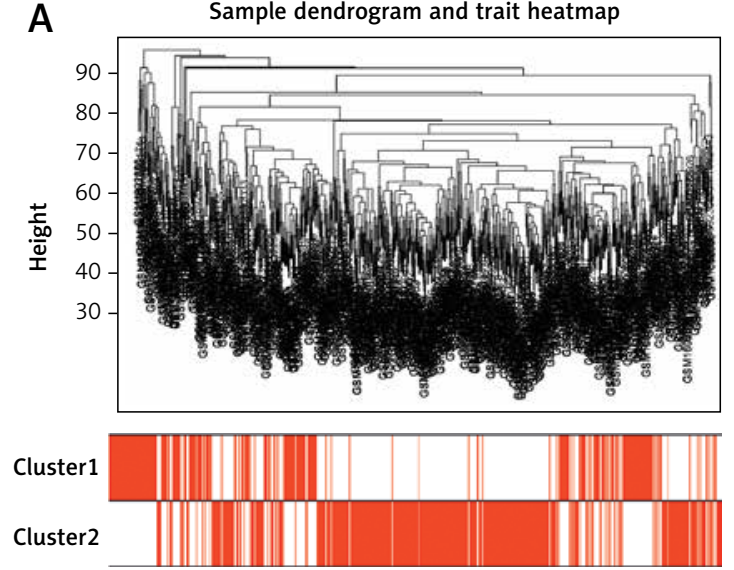

C
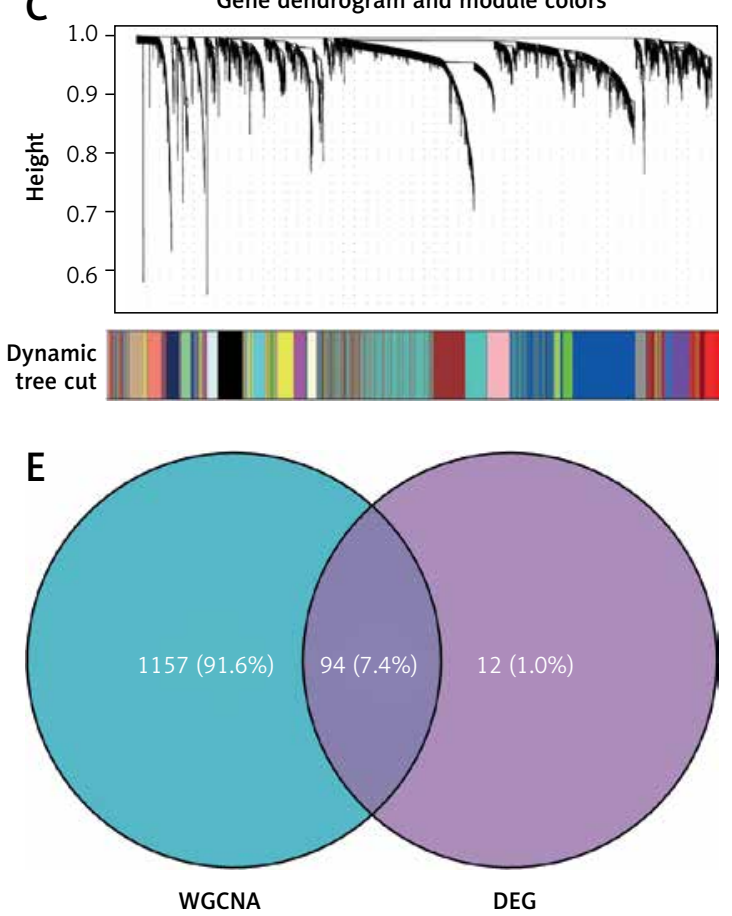

B
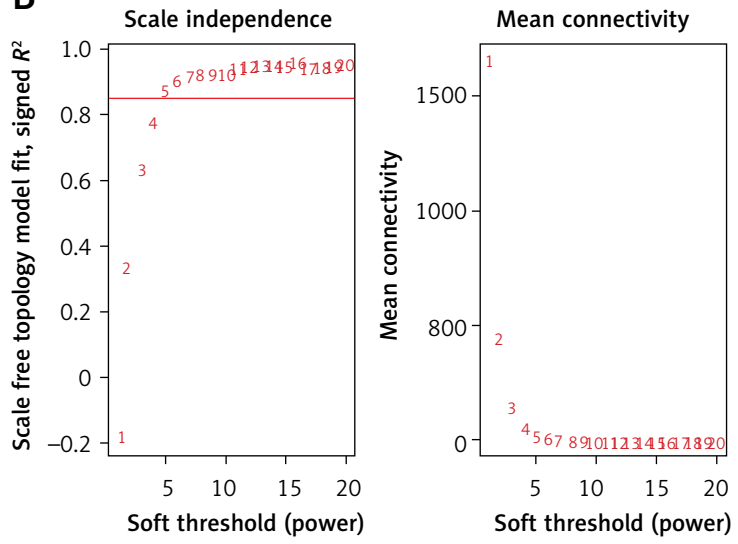

D

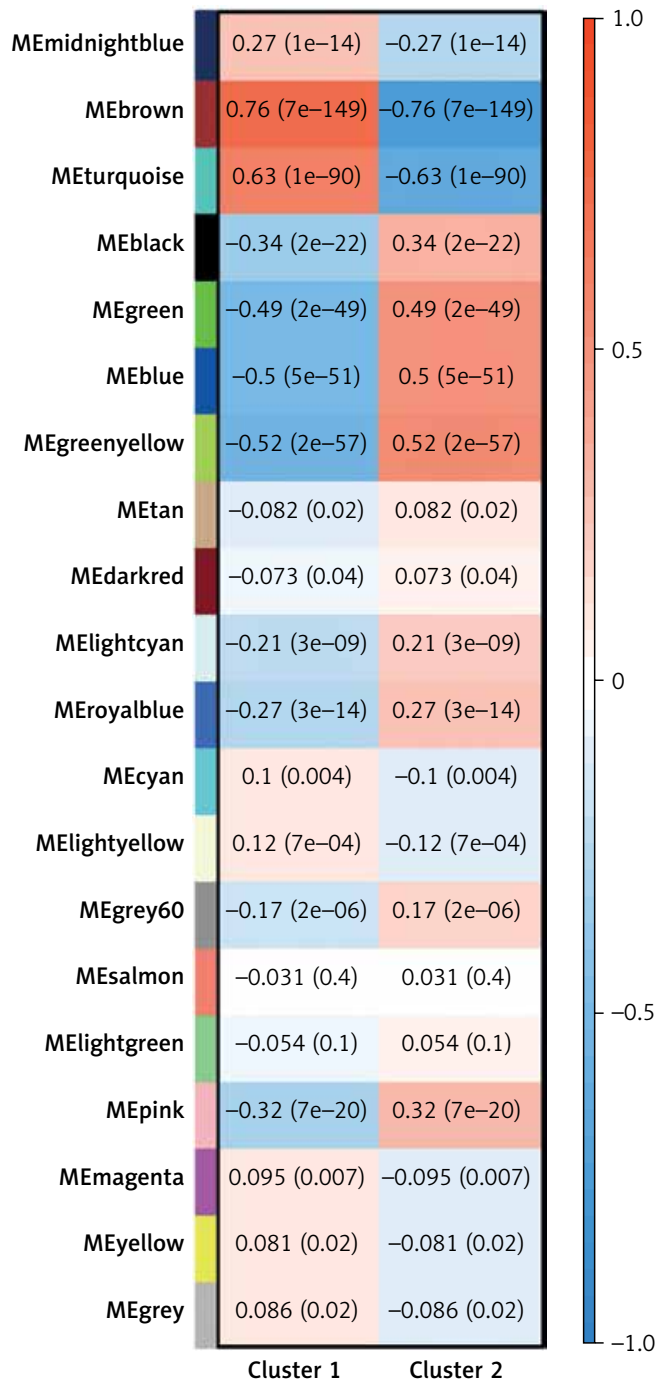

Figure 6. Construction of weighted co-expression network analysis. A - Clustering dendrogram based on Euclidean distance; B - Network topology analysis under various soft threshold powers. Left: The $x$-axis represents the soft threshold power. The $y$-axis represents the fit index of the scale-free topology model. Right: The $x$-axis represents the soft threshold power. The $y$-axis reflects the average connectivity (degrees); C - Cluster dendrograms of topologically overlapping genes with different similarities and assigned module colors; D - Module-trait association. Each row corresponds to a module, and each column corresponds to a feature. Each cell contains the corresponding correlation and $p$-value. This table is color-coded according to the relevance of the color legend; $\mathbf{E}-$ Venn diagram of the intersection of WGCNA's most relevant modular genes and differential genes 


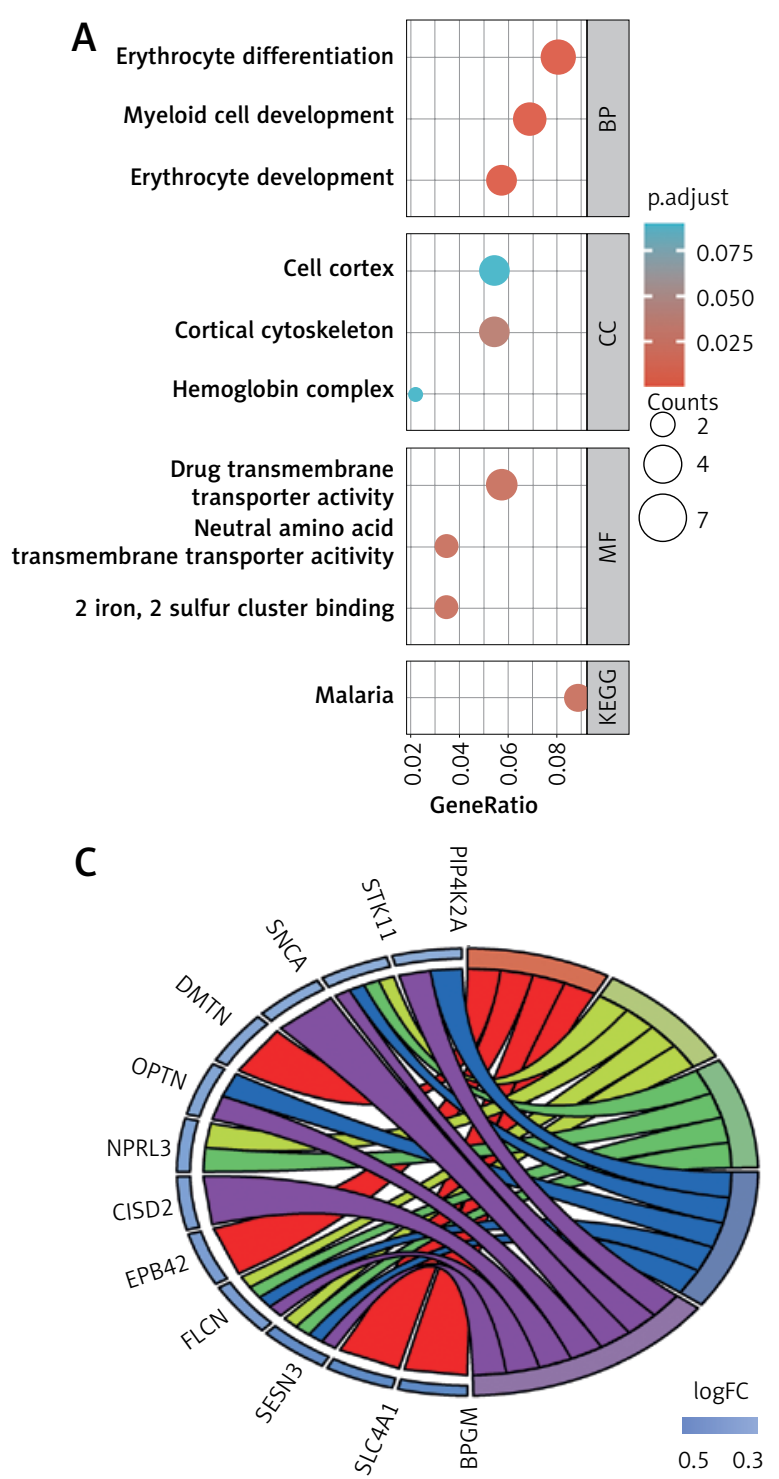

GO Terms Erythrocyte development $\square$ TORC1 signaling

negulation of autophagy Negative regulation of TOR signaling - Positive regulation of autophagy
B

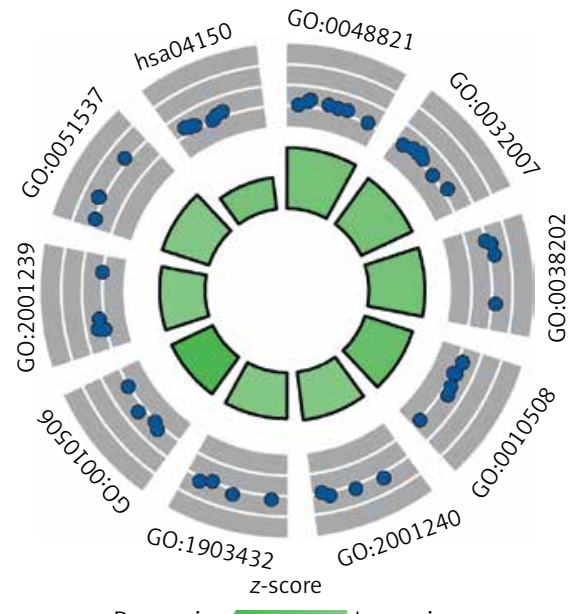

Decreasing Increasing

- $\operatorname{logFC}$ Downregulated

\begin{tabular}{|ll|}
\hline ID & \multicolumn{1}{c|}{ Description } \\
\hline GO:0048821 & Erythrocyte development \\
\hline GO:0032007 & Negative regulation of TOR signaling \\
\hline GO:0038202 & TORC1 signaling \\
\hline GO:0010508 & Positive regulation of autophagy \\
\hline GO:2001240 & $\begin{array}{l}\text { Negative regulation of extrinsic } \\
\text { apoptotic signaling pathway in absence }\end{array}$ \\
\hline GO:1903432 & Regulation of TORC1 signaling \\
\hline GO:0010506 & Regulation of autophagy \\
\hline GO:2001239 & $\begin{array}{l}\text { Regulation of extrinsic apoptotic } \\
\text { signaling pathway in absence of ligand }\end{array}$ \\
\hline GO:0051537 & 2 iron, 2 sulfur cluster binding \\
\hline hsa04150 & mTOR signaling pathway \\
\hline
\end{tabular}

D

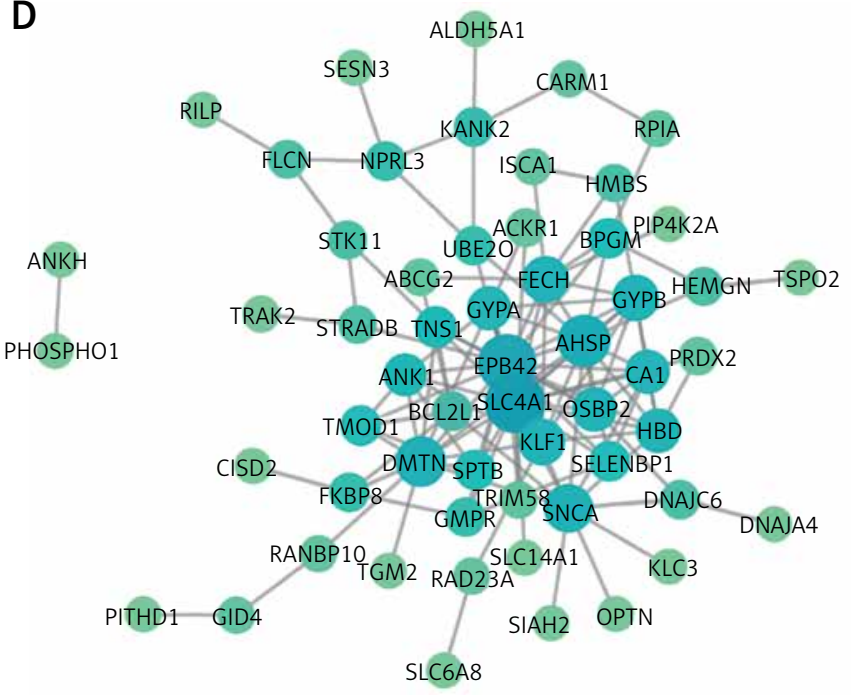

Figure 7. GO, KEGG enrichment analysis, and $\mathrm{PPI}$ network analysis. A - GO, KEGG analysis dot chart; B - GO, KEGG analysis circle chart, the center histogram represents the z-score score, dark green represents a decline, light green represents an increase, the middle dot represents genes, and blue represents down-regulated genes; C - Chord diagram of the selected $5 \mathrm{GO}$ paths; D - The PPI network of 62 common genes constructed by STRING database, the greater the degree value of the gene, the darker IGF2BP2 the color and the larger the diameter 
E
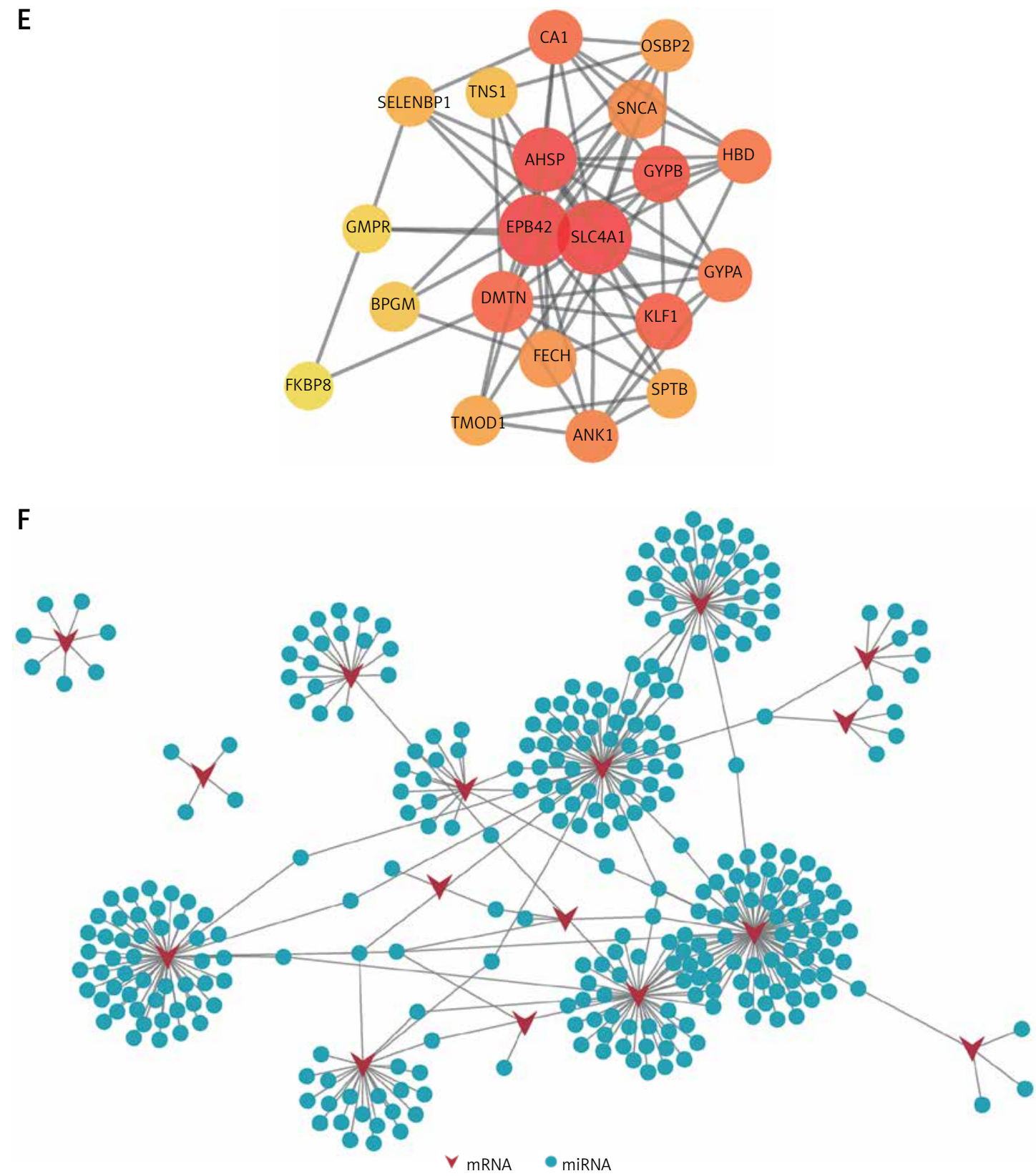

Figure 7. Cont. $\mathbf{E}$ - For the first 20 HUB genes calculated by cytoHubba, the higher the enrichment score, the darker the color; $\mathbf{F}-$ The miRNA-mRNA interaction network, the light blue circle represents miRNA, and the brown triangle represents mRNA

higher than that in the control group, and similar results were obtained in cell experiments (Figure $9 A-I)$.

\section{Drug sensitivity analysis}

Based on the IC50 of different cell lines for different small molecule drugs in the GDSC database, we analyzed changes in sensitivity of different ferroptosis classifications to these drugs. The results suggest that NU7441, AMG706, SL01011, GDC0941, sunitinib, metformin and other small molecule drugs have higher $\mathrm{IC}_{50}$ values in cluster2 than cluster1, and the difference is statistically significant $(p<0.001)$ (Figures $10 \mathrm{~A}-\mathrm{T})$. The results showed that a different classification of ferroptosis may lead to changes in the sensitivity of sepsis to 54 different small molecule drugs. The results showed that 20 small molecule drugs were statistically significant (Figures $10 \mathrm{~A}-\mathrm{T}$ ).

\section{Discussion}

Sepsis is a life-threatening medical emergency caused by a misregulated host response to inflammation in which microbial biological processes play an important role [6]. Iron is an important element in microbial biological processes, and many 
A

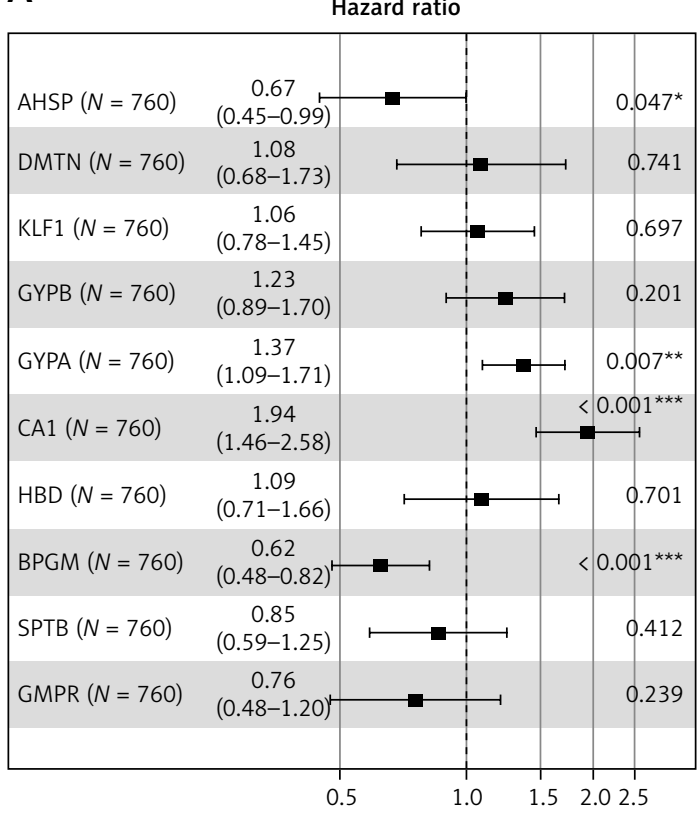

\#Events: 114; Global p-value (Log-Rank): 2.2730e-07, AIC: 1463.83 Concordance Index: 0.68 .

C

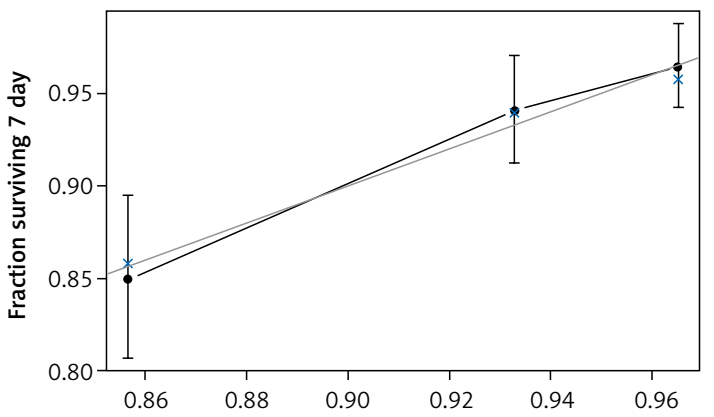

$N=760 d=114 p=10,250$ subjects per group, $X$ - resampling optimism added, $B=1000$, Gray: ideal. Based on observed-predicted

$\mathrm{E}$

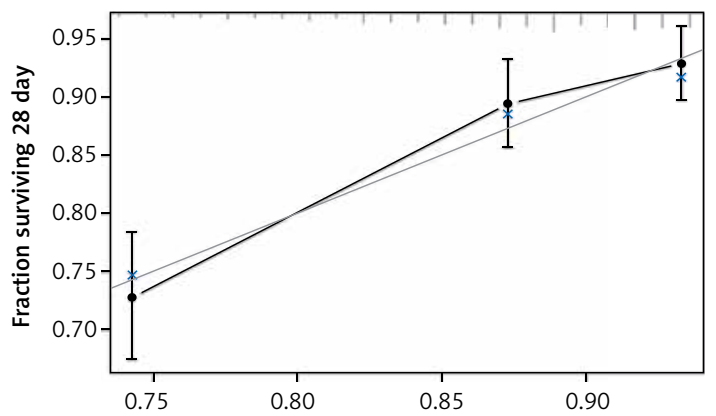

$N=760 d=114 p=10,250$ subjects per group, $X$ - resampling optimism added, $B=1000$, Gray: ideal. Based on observed-predicted

studies have revealed a relationship between changes in iron metabolism and sepsis [34]. Most microorganisms depend on iron for their pathogenicity, and some bacteria such as Escherichia coli and Klebsiella pneumoniae have evolved the ability to remove iron from host iron-binding proteins [35]. Previous studies have shown that iron
B

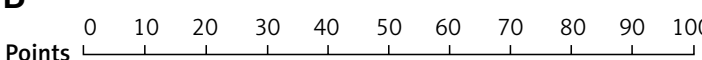

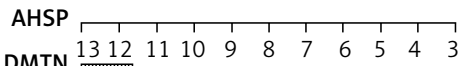

DMTN 3.58

KLF $\begin{array}{ccc}1 & \text { mाIIIITा } \\ 1 & 6 & 11\end{array}$

GYPB \begin{tabular}{lllllllll}
1 & 6 & 11 & & & & \\
\hline & 1 & 1 & 1 & 1 & 1 & 1 & 1
\end{tabular}

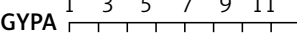

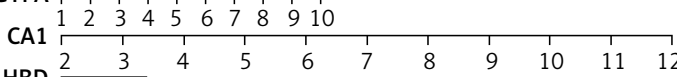

HBD $T$ TाTाTाTाTा

BPGM 25812

$\begin{array}{lllllllllllll}11 & 10 & 9 & 7 & 6 & 5 & 4 & 3 & 2 & 1\end{array}$

SPTB 7.553

GMPR $\begin{array}{llllllllllll}7.5 & 5 & 3 & & & & & & \\ 12 & 10 & 8 & 7 & 6 & 5 & 4 & 3 & 2\end{array}$

$\begin{array}{cccccccccccc}\text { Total } & 1 & 1 & 1 & 1 & 1 & 1 & 1 & 1\end{array}$

1-week survival

2-week survival

4-week survival

\begin{tabular}{|c|c|}
\hline 0.99 & 0.90 .80 .70 .5 \\
\hline 0.99 & 0.90 .80 .70 .50 .3 \\
\hline 0.99 & 0.90 .80 .60 .40 .2 \\
\hline
\end{tabular}

\section{D}

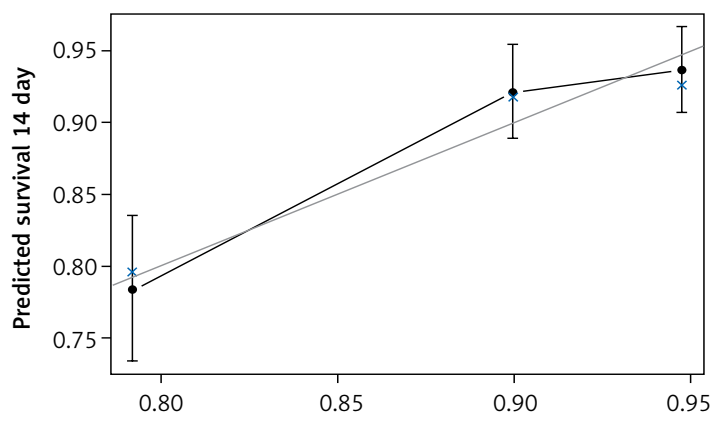

$N=760 d=114 p=10,250$ subjects per group, $X$ - resampling optimism added, $B=1000$, Gray: ideal. Based on observed-predicted

Figure 8. Correlation analysis of clinical features. A - Multi-factor Cox regression analysis forest plot; B - Multi-factor Cox regression analysis nomogram plot; C-E -7 days, 14 days, 28 days calibration curve

imbalance is associated with lower survival rates in patients with sepsis [36-38].

A number of studies have proposed that ferroptosis is a novel form of regulation of cell death closely related to iron overload [11, 18, 39]. Ferroptosis plays an important role in immune infiltration, but the specific mechanism remains un- 
Table III. GO, KEGG enrichment analysis results

\begin{tabular}{|lcccc|}
\hline ONTOLOGY & ID & Description & Count & $P$-value \\
\hline BP & GO:0048821 & Erythrocyte development & 5 & $4.19 \mathrm{E}-07$ \\
\hline BP & GO:0061515 & Myeloid cell development & 6 & $8.88 \mathrm{E}-07$ \\
\hline BP & GO:0030218 & Erythrocyte differentiation & 7 & $1.03 \mathrm{E}-06$ \\
\hline BP & GO:0038202 & TORC1 signaling & 4 & $4.72 \mathrm{E}-05$ \\
\hline BP & GO:0032007 & Negative regulation of TOR signaling & 4 & $9.30 \mathrm{E}-05$ \\
\hline BP & GO:0010506 & Regulation of autophagy & 8 & $1.43 \mathrm{E}-04$ \\
\hline BP & GO:0010508 & Positive regulation of autophagy & 5 & $2.18 \mathrm{E}-04$ \\
\hline BP & GO:1903432 & Regulation of TORC1 signaling & 3 & $6.77 \mathrm{E}-04$ \\
\hline BP & GO:2001240 & Negative regulation of extrinsic apoptotic signaling pathway in & 3 & $4.87 \mathrm{E}-04$ \\
& & absence of ligand & & \\
\hline BP & GO:2001239 & Regulation of extrinsic apoptotic signaling pathway in absence & 3 & $1.09 \mathrm{E}-03$ \\
& & of ligand & 5 & $2.11 \mathrm{E}-04$ \\
\hline CC & GO:0030863 & Cortical cytoskeleton & 2 & $1.38 \mathrm{E}-03$ \\
\hline CC & GO:0005833 & Hemoglobin complex & 5 & $1.71 \mathrm{E}-03$ \\
\hline CC & GO:0044448 & Cell cortex part & 5 & $8.98 \mathrm{E}-05$ \\
\hline MF & GO:0015238 & Drug transmembrane transporter activity & 3 & $1.65 \mathrm{E}-04$ \\
\hline MF & GO:0051537 & 2 iron, 2 sulfur cluster binding & 3 & $4.67 \mathrm{E}-04$ \\
\hline MF & GO:0015175 & Neutral amino acid transmembrane transporter activity & 4 & $1.61 \mathrm{E}-04$ \\
\hline KEGG & hsa05144 & Msa04150 & $1.05 \mathrm{E}-02$ \\
\hline KEGG & mor signaling pathway &
\end{tabular}

Table IV. Univariate and multivariate COX analysis results

\begin{tabular}{|c|c|c|c|c|c|c|}
\hline \multirow[t]{2}{*}{ Variables } & \multicolumn{3}{|c|}{ Univariate analysis } & \multicolumn{3}{|c|}{ Multivariate analysis } \\
\hline & $\mathrm{HR}$ & $95 \% \mathrm{Cl}$ of $\mathrm{HR}$ & $P$-value & $H R$ & $95 \% \mathrm{Cl}$ of $\mathrm{HR}$ & $P$-value \\
\hline Gender & 1.04 & $0.71-1.51$ & 0.832 & & & \\
\hline Age & 1.22 & $0.84-1.76$ & 0.284 & & & \\
\hline SLC4A1 & 1.08 & $0.94-1.22$ & 0.271 & & & \\
\hline EPB42 & 1.11 & $0.97-1.24$ & 0.109 & & & \\
\hline AHSP & 1.15 & $1.01-1.29$ & 0.029 & 0.67 & $0.44-0.99$ & 0.047 \\
\hline DMTN & 1.18 & $1.00-1.39$ & 0.046 & 1.08 & $0.67-1.72$ & 0.741 \\
\hline SNCA & 1.01 & $0.89-1.12$ & 0.923 & & & \\
\hline $\mathrm{FECH}$ & 1.06 & $0.95-1.18$ & 0.261 & & & \\
\hline KLF1 & 1.22 & $1.08-1.37$ & 0.001 & 1.06 & $0.78-1.44$ & 0.697 \\
\hline GYPB & 1.18 & $1.05-1.31$ & 0.003 & 1.23 & $0.89-1.7$ & 0.201 \\
\hline GYPA & 1.26 & $1.13-1.39$ & 0.000 & 1.37 & $1.09-1.71$ & 0.007 \\
\hline CA1 & 1.24 & $1.11-1.37$ & 0.000 & 1.94 & $1.45-2.58$ & 0.000 \\
\hline $\mathrm{HBD}$ & 1.18 & $1.05-1.31$ & 0.004 & 1.09 & $0.71-1.66$ & 0.701 \\
\hline OSBP2 & 1.09 & $0.94-1.24$ & 0.224 & & & \\
\hline ANK 1 & 1.11 & $0.98-1.24$ & 0.075 & & & \\
\hline TNS1 & 1.12 & $0.97-1.27$ & 0.106 & & & \\
\hline BPGM & 1.12 & $1.01-1.23$ & 0.018 & 0.62 & $0.47-0.81$ & 0.001 \\
\hline SELENBP1 & 1.06 & $0.94-1.18$ & 0.348 & & & \\
\hline TMOD1 & 1.07 & $0.93-1.22$ & 0.303 & & & \\
\hline SPTB & 1.22 & $1.02-1.46$ & 0.029 & 0.85 & $0.58-1.24$ & 0.412 \\
\hline FKBP8 & 1.17 & $0.98-1.39$ & 0.080 & & & \\
\hline GMPR & 1.16 & $1.00-1.32$ & 0.037 & 0.76 & $0.47-1.2$ & 0.239 \\
\hline
\end{tabular}


A

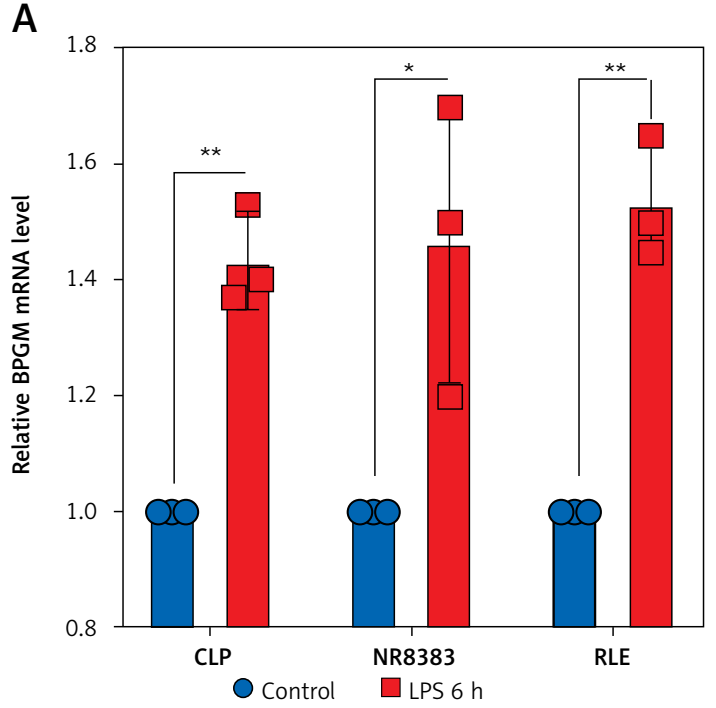

C

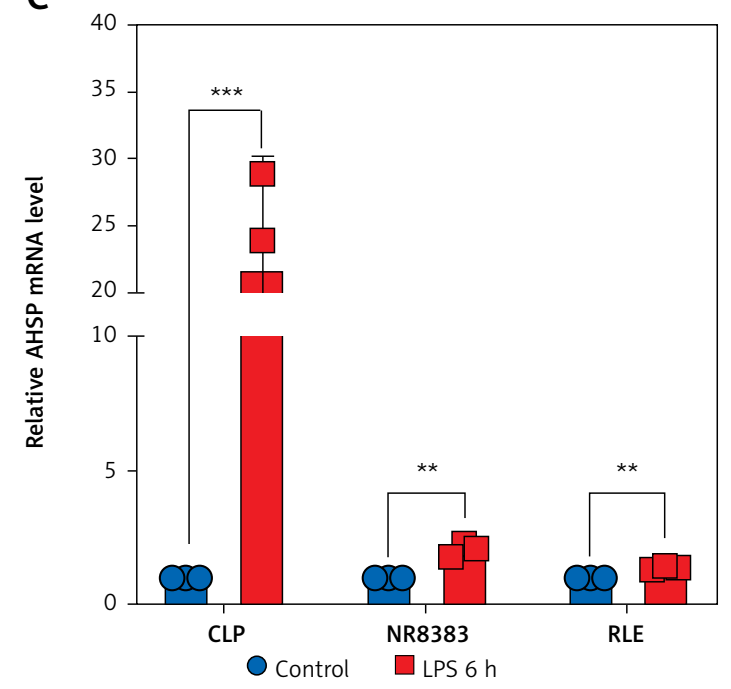

$E$

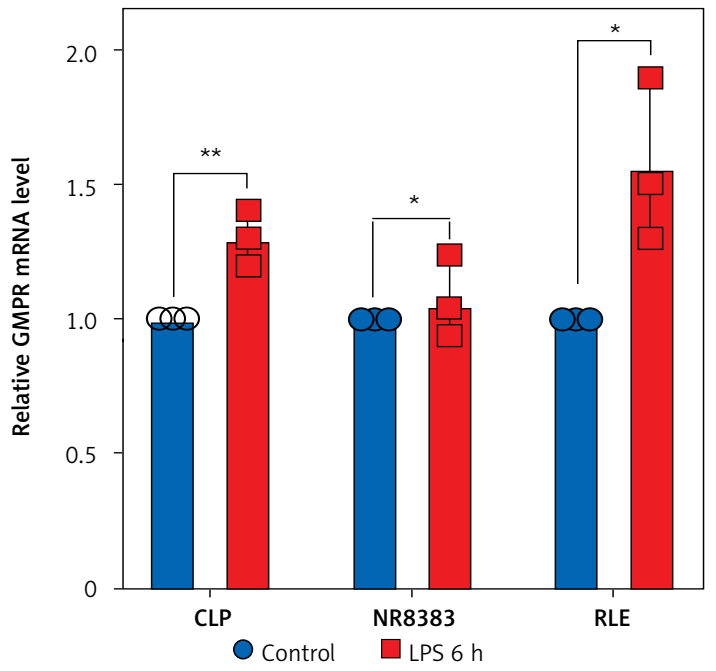

B

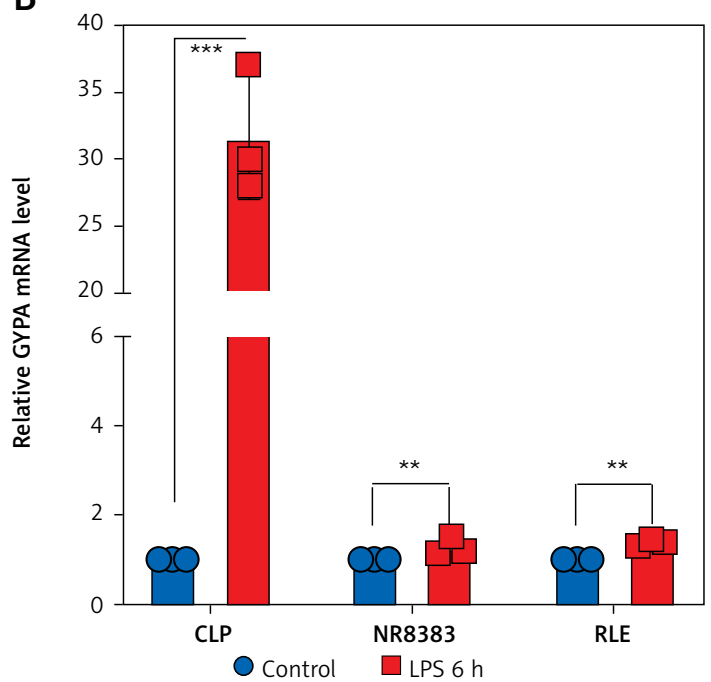

D

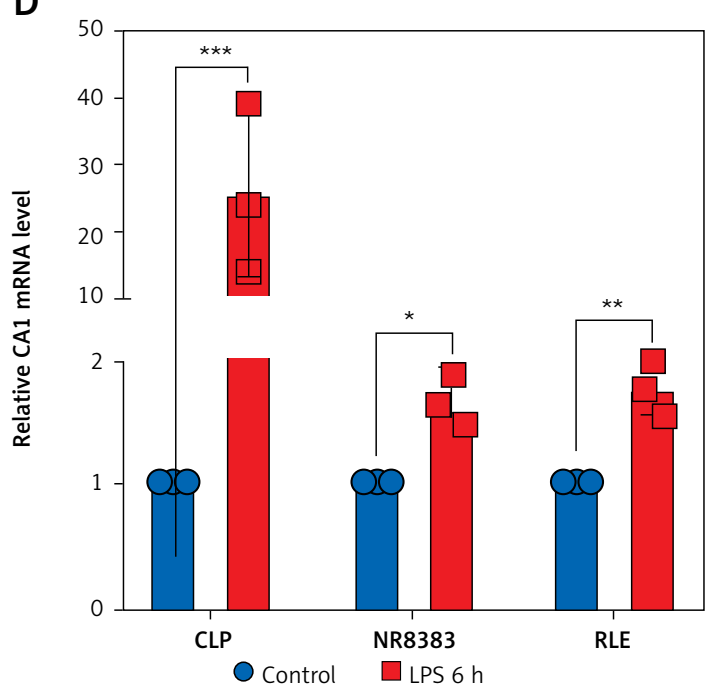

$\mathrm{F}$

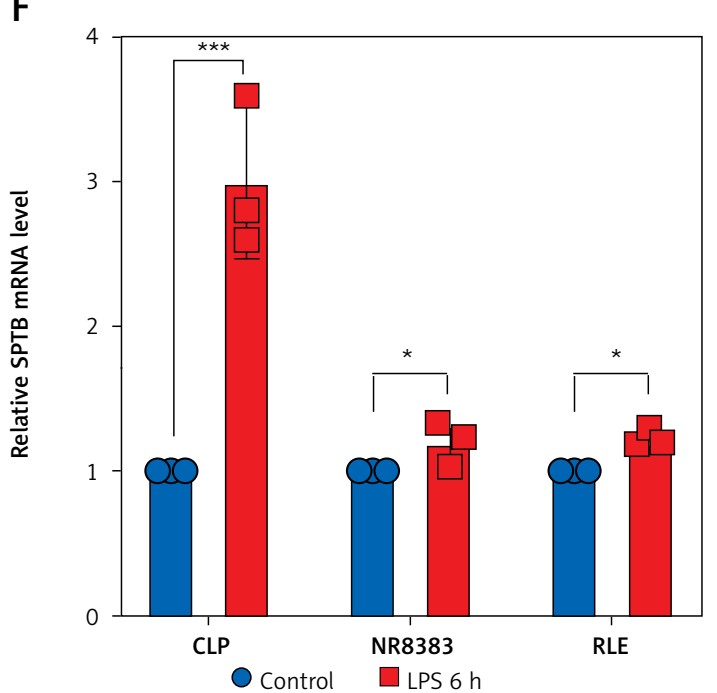

Figure 9. Expression of the genes in sepsis prognostic models increased in vivo and in vitro. The expression of BPGM (A), GYPA (B), AHSP (C), CA1 (D), GMPR (E), SPTB (F) 


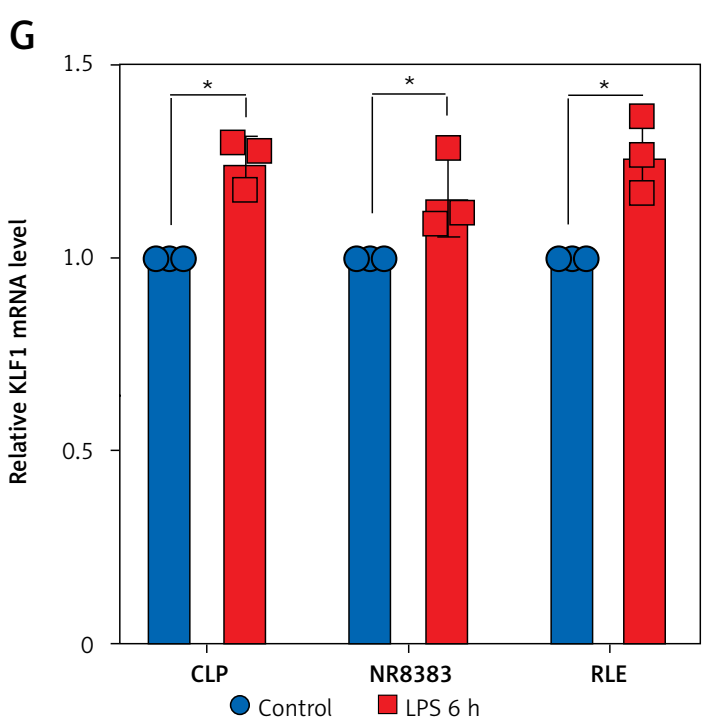

I

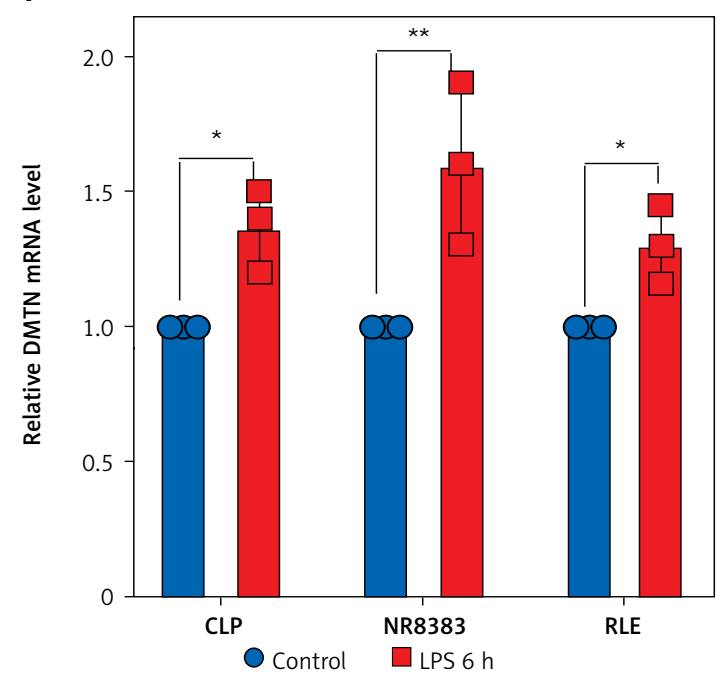

clear [40-42]. In this study, 10 ferroptosis-related genes were used to construct a sepsis prognostic model. Nine of these genes were validated in animal and cell models. Some of these genes have been shown to play different roles in immune infiltration. For example, the KLF1 gene is associated with immune activation [43]. Other genes may play an important role in ferroptosis. Sepsis is a highly heterogeneous disease. In the past, many studies have explored the biomarkers and prognostic models of sepsis, but these studies are limited to a single hematological index and do not comprehensively consider the level of gene expression. In addition, these predictive models are only valuable in one type of population, but have limited accuracy in another type of patient. Our prediction model is based on the expression of iron death-related genes, which is more accurate. The present prognostic model provides significantly better performance than previous prognostic models [44-46]. In addition, our nomogram

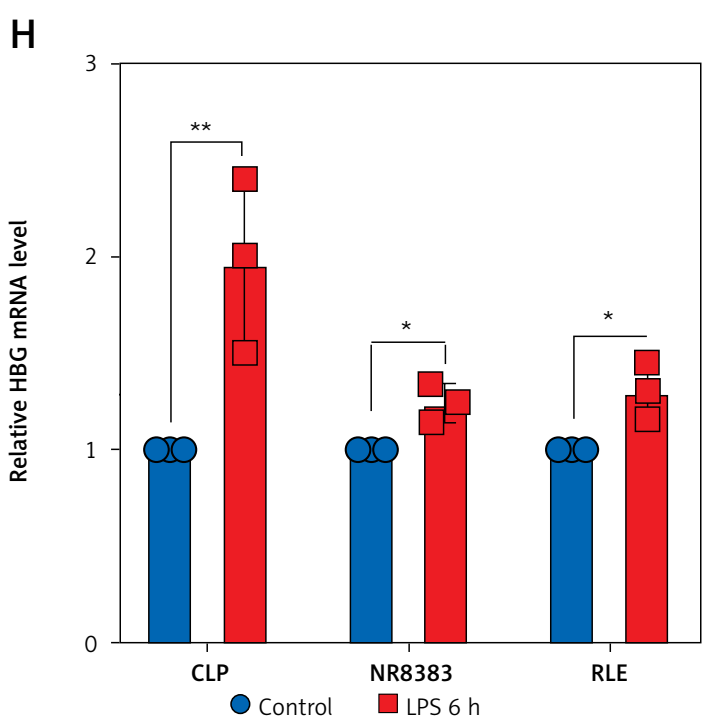

Figure 9. Cont. KLF1 (G), HBD (H) and DMTN (I) in the previous multivariate Cox regression model construction

can provide personalized prediction of mortality risk, which is of great significance to clinicians in clinical application.

In this study, we constructed subtypes based on the expression profile of ferroptosis genes to explore the biological differences among different types of ferroptosis subtypes of sepsis. Furthermore, 106 DEGs were studied and genes related to OS were screened out. Functional enrichment analysis showed that the DEGs were significantly correlated with inflammation, immunity and iron metabolism. The mechanism of inflammation susceptibility to ferroptosis has been a hot topic of research in the past decades, but the complex relationship between immunity and ferroptosis is still unknown [12, 47]. Studies have shown that inflammation can easily induce ferroptosis, and the activation of ferritin related pathways under inflammation may provide a new therapeutic target for the treatment of sepsis [12, 47]. We further explored the relationship between ferroptosis and 

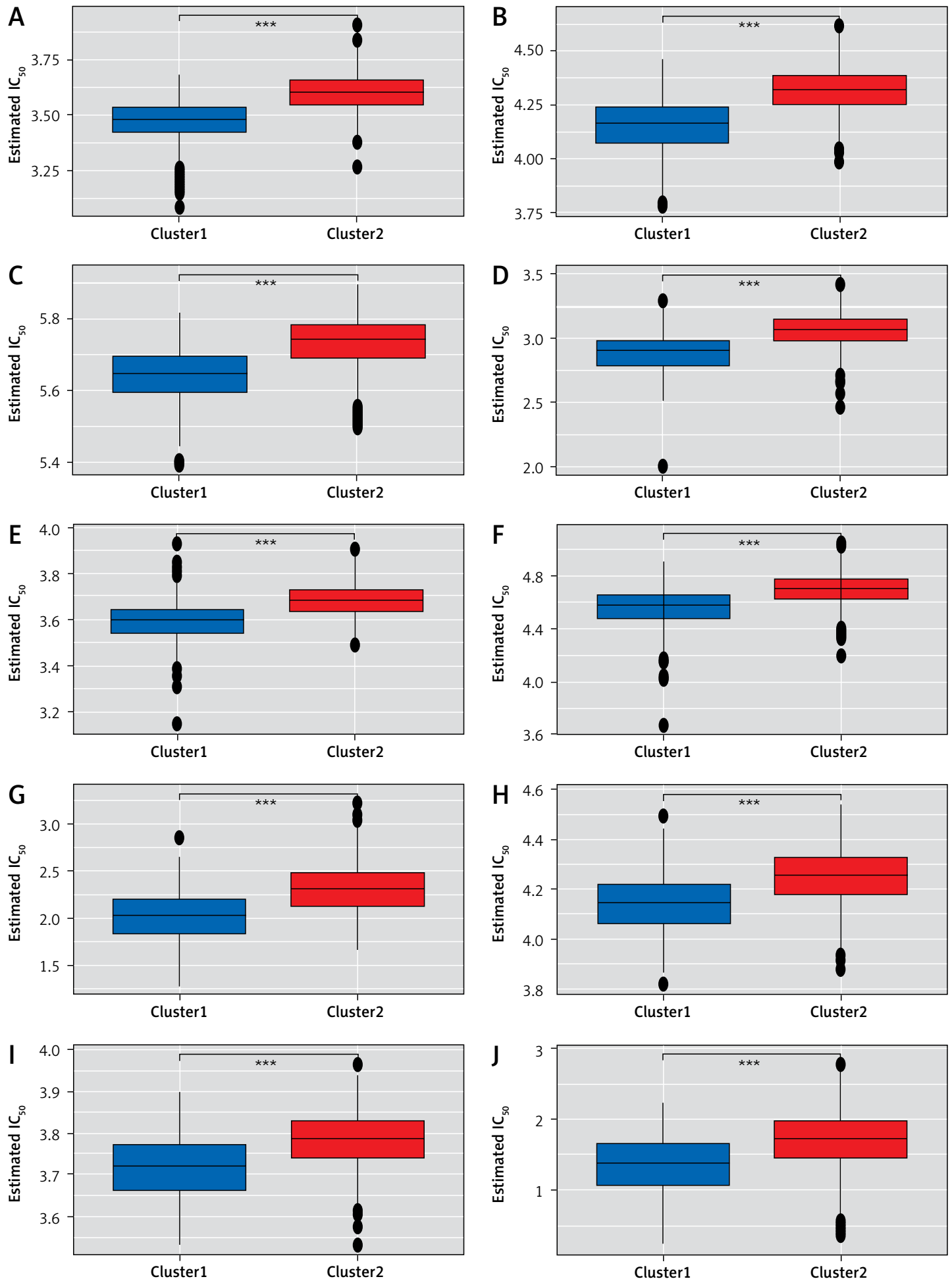

Figure 10. Sensitivity analysis of small molecule drugs. A-T - The sensitivity of different ferroptosis classifications to these small molecule drugs' changes. NU.7441 (A), AMG.706 (B), SL.0101.1 (C), W02009093972 (D), GSK269962A (E), EHT.1864 (F), GDC0941 (G), PD.173074 (H), sunitinib (I), NVP.TAE684 (J) 

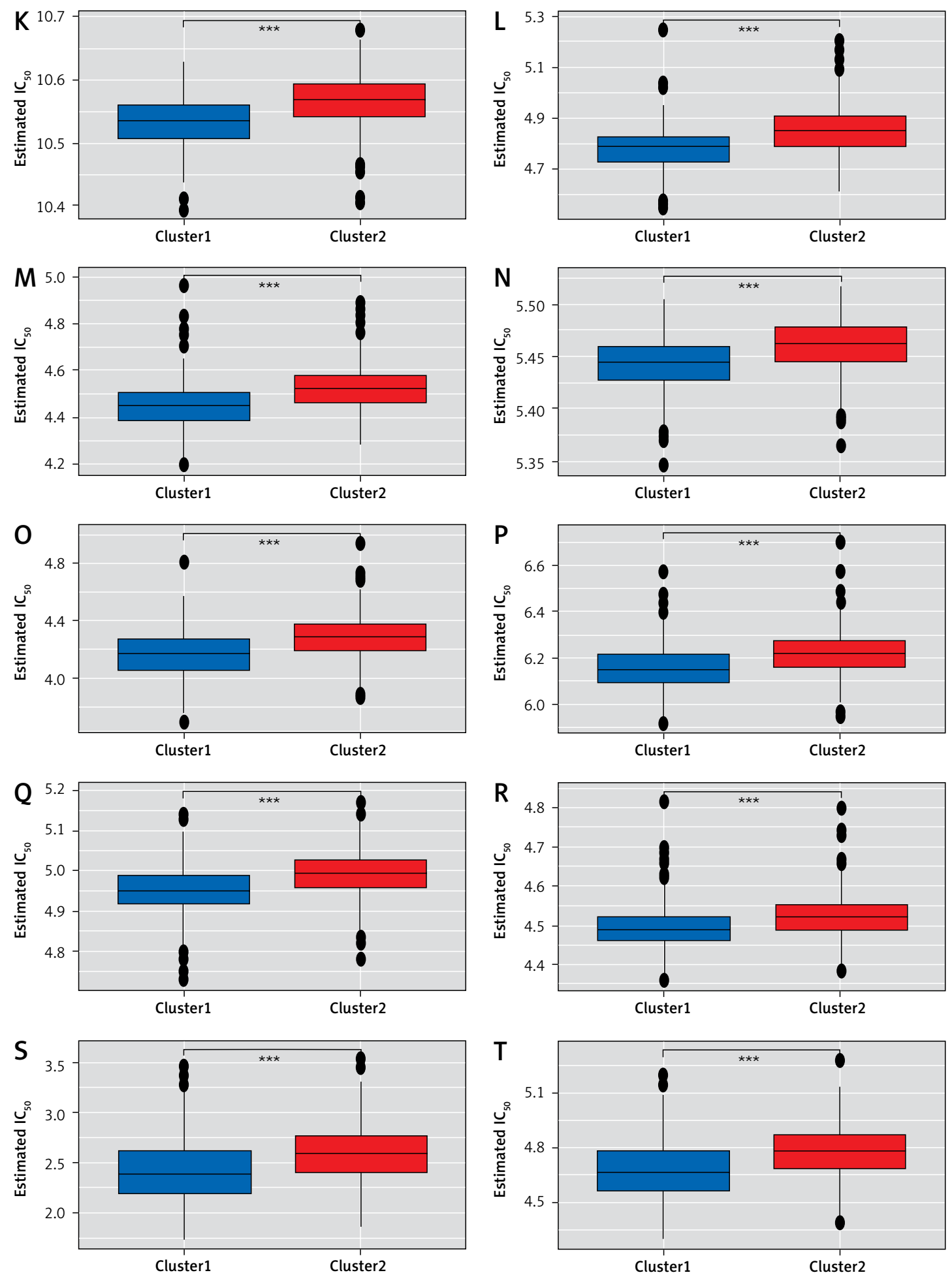

Figure 10. Cont. metformin (K), PHA.665752 (L), GNF.2 (M), ABT.888 (N), CGP.082996 (0), LFM.A13 (P), parthenolide (Q), PF.02341066 (R), BMS.536924 (S), bexarotene (T), etc. all had higher sensitivity in the cluster2 group 
immune cell infiltration. Notably, we found that ferroptosis significantly affected both B lymphocyte and CD8+ T cell function in the analysis of infiltrating immune cells. The correlation matrix of immune cells showed that the infiltration level of activated memory CD8+ T cells was highly correlated with neutrophil cells. However, we found that these results are somewhat similar to studies of features associated with ferroptosis in other diseases.

Through the GDSC database [48], based on the differential genes associated with ferroptosis, several small molecule drugs targeting the characteristic components associated with ferroptosis were identified. The results showed that 20 small molecule drugs were statistically significant. These small molecule drugs may provide a new therapeutic target for the treatment of sepsis.

There are some limitations to the study. Due to limited knowledge of ferroptosis, most of the characteristic components of our study involve not only ferroptosis-related pathways, but also other pathways. In addition, our research lacks validation of animal models. Our follow-up studies will further conduct IHC and FISH experiments on animal models of sepsis in order to provide pathological evidence. Moreover, because of our small sample size, it is difficult for our risk score to fully accurately assess the role of ferroptosis in sepsis. Therefore, our predictive model should be further validated in a cohort of sepsis from multicenter studies.

In conclusion, we identified two molecular subgroups by analyzing the expression of a matrix based on genes associated with ferroptosis in this study. The two molecular subgroups showed significantly different survival rates and immune status. Moreover, a sepsis prognostic model based on ferroptosis-related genes was developed and its predictive efficiency was well demonstrated. In addition, gene function enrichment analysis indicated that ferroptosis-related genes could affect the immune cell infiltration and then affect the occurrence, development and prognosis of sepsis. Our research indicated the important role of ferroptosis and immune interaction in the occurrence and development of sepsis, and provides a new idea for exploring the molecular mechanism and treatment of sepsis.

\section{Data availability statement}

The expression profile data and related clinical information were obtained from GEO databases (GSE65682, GSE95233). All relevant data and materials are available from the corresponding author upon reasonable request.

\section{Acknowledgments}

This work was supported by grants from the National Natural Science Foundation of China
(Nos.81871548-82160363). This work was also supported by The Featured Clinical Discipline Project of Shanghai Pudong (PWYst2018-01) and Key Discipline Group Construction Project of Shanghai Pudong (PWZxq2017-02).

Wei Dai and Tian Yu contributed equally to this work. Senior author: Ping Zheng (jojo_ras@126. com)

\section{Conflict of interest}

The authors declare no conflict of interest.

\section{References}

1. Cecconi M, Evans L, Levy M, Rhodes A. Sepsis and septic shock. Lancet 2018; 392: 75-87.

2. Jedynak M, Siemiatkowski A, Milewski R, Mroczko B, Szmitkowski M. Diagnostic effectiveness of soluble triggering receptor expressed on myeloid cells-1 in sepsis, severe sepsis and septic shock. Arch Med Sci 2019; 15: 713-21.

3. Faix JD. Biomarkers of sepsis. Crit Rev Clin Labor Sci 2013; 50: 23-36.

4. Minasyan H. Sepsis: mechanisms of bacterial injury to the patient. Scand I Trauma Resuscitation Emerg Med 2019; 27: 19.

5. Hawiger J, Veach RA, Zienkiewicz J. New paradigms in sepsis: from prevention to protection of failing microcirculation. J Thrombosis Haemostasis 2015; 13: 1743-56.

6. Nolt B, Tu F, Wang X, et al. Lactate and immunosuppression in sepsis. Shock 2018; 49: 120-5.

7. Zheng Z, Ma H, Zhang X, et al. Enhanced glycolytic metabolism contributes to cardiac dysfunction in polymicrobial sepsis. J Infect Dis 2017; 215: 1396-406.

8. Cheng SC, Scicluna BP, Arts RJ, et al. Broad defects in the energy metabolism of leukocytes underlie immunoparalysis in sepsis. Nature Immunol 2016; 17: 406-13.

9. Stockwell BR, Jiang X, Gu W. Emerging mechanisms and disease relevance of ferroptosis. Trends Cell Biol 2020; 30: 478-90.

10. Li J, Cao F, Yin HL, et al. Ferroptosis: past, present and future. Cell Death Disease 2020; 11: 88.

11. Zhou B, Liu J, Kang R, Klionsky DJ, Kroemer G, Tang D. Ferroptosis is a type of autophagy-dependent cell death. Semin Cancer Biol 2020; 66: 89-100.

12. Sun $\mathrm{Y}$, Chen $\mathrm{P}$, Zhai B, et al. The emerging role of ferroptosis in inflammation. Biomed Pharmacother 2020; 127: 110108

13. Park E, Chung SW. ROS-mediated autophagy increases intracellular iron levels and ferroptosis by ferritin and transferrin receptor regulation. Cell Death Dis 2019; 10: 822

14. Hirschhorn T, Stockwell BR. The development of the concept of ferroptosis. Free Radical Biol Med 2019; 133: 130-43.

15. Hadian K, Stockwell BR. SnapShot: ferroptosis. Cell 2020; 181: 1188-1188.e1.

16. Feng H, Schorpp K, Jin J, et al. Transferrin receptor is a specific ferroptosis marker. Cell Reports 2020; 30: 3411-23.e7.

17. Li N, Wang W, Zhou H, et al. Ferritinophagy-mediated ferroptosis is involved in sepsis-induced cardiac injury. Free Radical Biol Med 2020; 160: 303-18.

18. Qiu Y, Cao Y, Cao W, Jia Y, Lu N. The application of ferroptosis in diseases. Pharmacol Res 2020; 159: 104919. 
19. Shan Y, Yang G, Huang H, et al. Ubiquitin-like modifier activating enzyme 1 as a novel diagnostic and prognostic indicator that correlates with ferroptosis and the malignant phenotypes of liver cancer cells. Front Oncol 2020; 10: 592413.

20. Liu Y, Zhang X, Zhang J, Tan J, Li J, Song Z. Development and validation of a combined ferroptosis and immune prognostic classifier for hepatocellular carcinoma. Front Cell Develop Biol 2020; 8: 596679.

21. Liang JY, Wang DS, Lin HC, et al. A novel ferroptosis-related gene signature for overall survival prediction in patients with hepatocellular carcinoma. Int J Biol Sci 2020; 16: 2430-41.

22. Wang F, Lv H, Zhao B, et al. Iron and leukemia: new insights for future treatments. J Exp Clin Cancer Res 2019; 38: 406.

23. Scicluna BP, Klein Klouwenberg PM, van Vught LA, et al. A molecular biomarker to diagnose community-acquired pneumonia on intensive care unit admission. Am J Respir Crit Care Med 2015; 192: 826-35.

24. Tabone O, Mommert M, Jourdan C, et al. Endogenous retroviruses transcriptional modulation after severe infection, trauma and burn. Front Immunol 2018; 9: 3091

25. Davis S, Meltzer PS. GEOquery: a bridge between the Gene Expression Omnibus (GEO) and BioConductor Bioinformatics 2007; 23: 1846-7.

26. Wilkerson MD, Hayes DN. ConsensusClusterPlus: a class discovery tool with confidence assessments and item tracking. Bioinformatics 2010; 26: 1572-3.

27. Ritchie ME, Phipson B, Wu D, et al. limma powers differential expression analyses for RNA-sequencing and microarray studies. Nucleic Acids Res 2015; 43: e47.

28. Ito K, Murphy D. Application of ggplot2 to Pharmacometric Graphics. CPT Pharmacometrics Systems Pharmacology 2013; 2: e79.

29. Newman AM, Liu CL, Green MR, et al. Robust enumeration of cell subsets from tissue expression profiles. Nature Methods 2015; 12: 453-7.

30. Subramanian A, Tamayo P, Mootha VK, et al. Gene set enrichment analysis: a knowledge-based approach for interpreting genome-wide expression profiles. Proc Natl Acad Sci USA 2005; 102: 15545-50.

31. Liang JW, Fang ZY, Huang Y, et al. Application of weighted gene co-expression network analysis to explore the key genes in Alzheimer's disease. J Alzheimer's Dis 2018; 65: 1353-64.

32. Yu G, Wang LG, Han Y, He QY. clusterProfiler: an R package for comparing biological themes among gene clusters. OMICS 2012; 16: 284-7.

33. Lu X, Jiang L, Zhang L, et al. Immune signature-based subtypes of cervical squamous cell carcinoma tightly associated with human papillomavirus type 16 expression, molecular features, and clinical outcome. Neoplasia 2019; 21: 591-601.

34. Liu Q, Wu J, Zhang X, Wu X, Zhao Y, Ren J. Iron homeostasis and disorders revisited in the sepsis. Free Radical Biol Med 2021; 165: 1-13.

35. Ganz T, Nemeth E. Iron homeostasis in host defence and inflammation. Nature Rev Immunol 2015; 15: 500-10.

36. Fedotcheva N, Olenin A, Beloborodova N. Influence of microbial metabolites on the nonspecific permeability of mitochondrial membranes under conditions of acidosis and loading with calcium and iron ions. Biomedicines 2021; 9: 558.

37. Fan H, Zhao Y, Sun M, Zhu JH. Urinary neutrophil gelatinase-associated lipocalin, kedney injury molecule-1, $\mathrm{N}$-acetyl- $\beta$-D-glucosaminidase levels and mortality risk in septic patients with acute kidney injury. Arch Med Sci 2018; 14: 1381-6.

38. Jung M, Drapier JC, Weidenbach $\mathrm{H}$, et al. Effects of hepatocellular iron imbalance on nitric oxide and reactive oxygen intermediates production in a model of sepsis. J Hepatol 2000; 33: 387-94.

39. Zhu J, Xiong Y, Zhang Y, et al. The molecular mechanisms of regulating oxidative stress-induced ferroptosis and therapeutic strategy in tumors. Oxid Med Cellular Longev 2020; 2020: 8810785.

40. Mou Y, Wu J, Zhang Y, Abdihamid O, Duan C, Li B. Low expression of ferritinophagy-related NCOA4 gene in relation to unfavorable outcome and defective immune cells infiltration in clear cell renal carcinoma. BMC Cancer 2021; 21: 18.

41. Yu B, Choi B, Li W, Kim DH. Magnetic field boosted ferroptosis-like cell death and responsive MRI using hybrid vesicles for cancer immunotherapy. Nature Commun 2020; 11: 3637

42. Tang $\mathrm{R}$, Hua J, Xu J, et al. The role of ferroptosis regulators in the prognosis, immune activity and gemcitabine resistance of pancreatic cancer. Ann Transl Med 2020; 8: 1347.

43. Teruya S, Okamura T, Komai T, et al. Egr2-independent, Klf1-mediated induction of PD-L1 in CD4(+) T cells. Sci Rep 2018; 8: 7021.

44. Zhang Z, Pan Q, Ge H, Xing L, Hong Y, Chen P. Deep learning-based clustering robustly identified two classes of sepsis with both prognostic and predictive values. EBioMedicine 2020; 62: 103081.

45. Weber GF, Chousterman BG, He S, et al. Interleukin-3 amplifies acute inflammation and is a potential therapeutic target in sepsis. Science 2015; 347: 1260-5.

46. Sandquist M, Wong HR. Biomarkers of sepsis and their potential value in diagnosis, prognosis and treatment. Exp Rev Clin Immunol 2014; 10: 1349-56.

47. Su LJ, Zhang JH, Gomez H, et al. Reactive oxygen species-induced lipid peroxidation in apoptosis, autophagy, and ferroptosis. Oxid Med Cellular Longev 2019; 2019: 5080843.

48. Yang W, Soares J, Greninger P, et al. Genomics of Drug Sensitivity in Cancer (GDSC): a resource for therapeutic biomarker discovery in cancer cells. Nucleic Acids Res 2013: 41: D955-61. 